TITLE:

\title{
Energetics of the single-headed kinesin KIF1A
}

$\operatorname{AUTHOR}(\mathrm{S})$ :

Kanada, Ryo; Sasaki, Kazuo

CITATION:

Kanada, Ryo ...[et al]. Energetics of the single-headed kinesin KIF1A. Physical Review E 2013, 88(2): 022711.

ISSUE DATE:

2013-08-14

URL:

http://hdl.handle.net/2433/179284

RIGHT:

(C)2013 American Physical Society 
PHYSICAL REVIEW E 88, 022711 (2013)

\title{
Energetics of the single-headed kinesin KIF1A
}

\author{
Ryo Kanada* \\ Cybermedia Center, Osaka University, Toyonaka 560-0043, Japan \\ and Department of Biophysics Graduate School of Science, Kyoto University, Kyoto 606-8502, Japan
}

Kazuo Sasaki

Department of Applied Physics, Tohoku University, 6-6-05 Aoba-yama, Sendai 980-8579, Japan

(Received 31 October 2012; revised manuscript received 10 June 2013; published 14 August 2013)

\begin{abstract}
KIF1A is a single-headed molecular motor that moves processively and unidirectionally along a microtubule by using the chemical energy released by hydrolyzing adenosine triphosphate (ATP) into adenosine diphosphate (ADP) and inorganic phosphate $\left(\mathrm{P}_{\mathrm{i}}\right)$. Although the movement of KIF1A seems to have successfully been explained by a simple Brownian motor model of the flashing ratchet type, this model is not suited to discuss the energetics of KIF1A. We introduce an elaborated model of the ratchet type to investigate how the chemical free energy is converted into mechanical work by taking account of the binding and release of reactant (ATP) and product (ADP and $\mathrm{P}_{\mathrm{i}}$ ) molecules to and from the motor. The efficiency of energy transduction, the power output, and other quantities are calculated from the analytically obtained steady-state solution of the Fokker-Planck equations. It turns out that the concentrations of the reactant and product molecules that optimize both the efficiency and the power are close to those in the cell.
\end{abstract}

DOI: 10.1103/PhysRevE.88.022711

PACS number(s): 87.16.Nn, 87.10.-e, 05.40.-a

\section{INTRODUCTION}

Biological molecular motors play crucial roles in cell activities such as intracellular transport, muscle contraction, and cell division [1-3]. Among others, KIF1A is a unique motor protein. It is a member of the kinesin-3 family in the kinesin superfamily [4]. Motors in this superfamily move along a linear track called a microtubule to transport cargoes such as membranous organelles and protein complexes in cells [5]. The energy necessary for the movement is supplied by a chemical reaction in which adenosine triphosphate (ATP) is hydrolyzed to adenosine diphosphate (ADP) and inorganic phosphate $\left(\mathrm{P}_{\mathrm{i}}\right)$. Most motors in the kinesin superfamily are dimers and have two "heads," which interact with the microtubule. They move in a "hand-over-hand" fashion by using their heads just as we use our legs when we walk. At least one head can hold a microtubule to prevent a dimeric kinesin from being detached and diffusing away from the microtubule. By contrast, KIF1A is a monomer and has only one head. Nevertheless, it can move along a microtubule processively (without being fully detached) more than $1 \mu \mathrm{m}$ [6]. The mechanism of how a singleheaded kinesin moves has not been completely understood.

It has been recognized that certain fundamental features of biological molecular motors may be captured by "Brownian motor" or ratchet models [7-11]. Okada and Hirokawa [6,12] demonstrated that a two-state (flashing) ratchet model can be used to analyze their experiments on the motility of KIF1A. In this model, the motor is weakly bound to the microtubule in one state and it is strongly bound in the other state. The switching between the states is triggered by certain chemical changes in the motor and it occurs stochastically. The motor undergoes diffusive motion in the weak-binding state and it is subject to an asymmetric, periodic potential of a sawtooth shape in the strong-binding state. The asymmetry of the potential causes a

*kanada@theory.biophys.kyoto-u.ac.jp displacement of the motor biased toward one direction upon the transition from the weak-binding state to the strong-binding state. This biased displacement leads to the unidirectional motion of the motor. Although the simple two-state ratchet model is successful in explaining the observed motion of KIF1A even in the presence of external load [13], it does not take into account the effects of the concentrations of ATP, ADP, and $\mathrm{P}_{\mathrm{i}}$, which affect the amount of energy liberated by the ATP hydrolysis and the rates of changes in chemical states of the motor. In order to reveal how well the motor performs from the energetic point of view and to clarify if the concentrations of ATP, ADP, and $\mathrm{P}_{\mathrm{i}}$ in cells are optimal for the performance of the motor, we need to study the dependence of the efficiency of energy transduction and other properties on these concentrations.

The purpose of the present paper is to improve the ratchettype model for KIF1A and investigate the issues raised above. Following the idea presented in Ref. [14], we modify the ratchet model in such a way that a transition between states occurs when a reactant or product molecule is attached to or detached from the motor. This implies that we need three states instead of two, because three kinds of molecules (ATP, ADP, and $\mathrm{P}_{\mathrm{i}}$ ) are involved in the ATP hydrolysis. We keep the model so simple that analytic results for the average velocity of the motor and the reaction rate can be obtained. These results are used to study the dependence of the energetic properties of the motor (the thermodynamic efficiency, the power output, and a so-called generalized efficiency $[15,16])$ on the concentrations of ATP, ADP, and $\mathrm{P}_{\mathrm{i}}$. We find that the concentrations of these molecules in cells more or less optimize these indices of KIF1A's performance simultaneously. The present model also provides the information on whether the motor synthesize ATP when it is forced to move backward; it turns out that the ATP synthesis is possible in principle but the condition for it is unlikely to be realized in practice.

The paper is organized as follows. In Sec. II, our model is described and the analytic results for the average velocity 
and the reaction rate are presented. In Sec. III, the dependence of the reaction rate on the concentrations of ATP, ADP, and $\mathrm{P}_{\mathrm{i}}$ are analyzed and the values of the model parameters are determined from the comparison with experiments of KIF1A and other means. In Sec. IV, the dependence of the velocity of the motor on the load force as well as the concentrations of ATP, ADP, and $\mathrm{P}_{\mathrm{i}}$ is discussed. The possibility of ATP synthesis by KIF1A is also discussed in this section. In Sec. V, the efficiency of energy transduction, the power output, and the generalized efficiency are studied. Section VI provides a brief conclusion. Mathematical details of some calculations are given in Appendices A-E.

\section{MODEL}

\section{A. Background}

KIF1A is a molecular motor that catalyzes the ATP hydrolysis reaction,

$$
\mathrm{ATP} \rightarrow \mathrm{ADP}+\mathrm{P}_{\mathrm{i}},
$$

and moves along the microtubule (MT) unidirectionally and processively [12]. In a cycle of the chemical reaction (1), KIF1A goes through four "chemical states" as $\Phi \rightarrow \mathrm{T} \rightarrow$ $\mathrm{D} \cdot \mathrm{P} \rightarrow \mathrm{D} \rightarrow \Phi$, where $\Phi$ represents the motor with no nucleotide (ATP, ADP, and $\mathrm{P}_{\mathrm{i}}$ ) bound, $\mathrm{T}$ the motor with ATP bound, D.P the motor with ADP and $\mathrm{P}_{\mathrm{i}}$ bound, and D the motor with ADP bound.

It is known that KIF1A is bound weakly to the MT and undergoes free diffusion along it in state $\mathrm{D}$, whereas it is strongly bound to the MT and does not move in the other states [12]. It was observed that KIF1A moves to the plus end of the MT upon the transition $\mathrm{D} \rightarrow \Phi$ by a distance of about $3 \mathrm{~nm}$ [13], and this "biased binding" is believed to be the main mechanism of the unidirectional motion of KIF1A.

One of the simplest models to account for the observations explained above is the two-state (flashing) ratchet model. In this model, the motor undergoes the free Brownian motion along the track in one state (weak-binding state), and a periodic, asymmetric potential $V_{0}(x)$ shown in Fig. 1 acts on the motor in the other state (strong-binding state), where $x$ is the position of the motor along the track. Potential $V_{0}(x)$ is defined by

$$
V_{0}(x)= \begin{cases}W x / a & (0 \leqslant x<a) \\ W(L-x) /(L-a) & (a \leqslant x<L)\end{cases}
$$

in the interval $(0, L)$ and its period is $L$, where $a$ is a constant satisfying $0<a<L / 2$. This potential represents the interaction between the motor and the track, and it reflects the periodicity ( $L=8 \mathrm{~nm}$ for the MT) and asymmetric (polar) structure of the track. Repeated transitions between these states

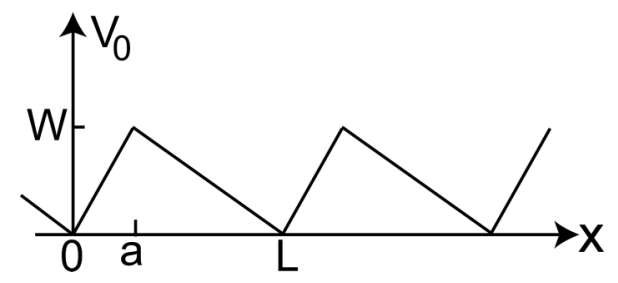

FIG. 1. The sawtooth potential defined in Eq. (2). results in a net motion toward the direction of increasing $x$ due to the rectification of the Brownian motion by the asymmetric potential [8]. A transition from the strong-binding state to the weak-binding state is usually assumed to be triggered by the chemical reaction (1) or by the thermal fluctuation [17]. In this scheme the chemical reaction is taken into account only through

$$
\Delta \mu \equiv \mu_{\mathrm{T}}-\mu_{\mathrm{D}}-\mu_{\mathrm{P}},
$$

where $\mu_{\mathrm{T}}, \mu_{\mathrm{D}}$, and $\mu_{\mathrm{P}}$ are the chemical potentials of ATP, $\mathrm{ADP}$, and $\mathrm{P}_{\mathrm{i}}$, respectively. However, the reaction rate and the velocity of the motor should depend on $\mu_{\mathrm{T}}, \mu_{\mathrm{D}}$, and $\mu_{\mathrm{P}}$ (i.e., the concentrations of ATP, ADP, and $\mathrm{P}_{\mathrm{i}}$ ) separately, not only through the particular combination in Eq. (3).

\section{B. Outline of the model}

We would like to modify the ratchet model explained above in such a way that the effects of $\mu_{\mathrm{T}}, \mu_{\mathrm{D}}$, and $\mu_{\mathrm{P}}$ can be taken into account separately by following the prescription presented in Ref. [14]. A minimal model satisfying this requirement seems to be a model with three internal states, which we call states $\Phi, \mathrm{T}$, and D. Here, states $\Phi$ and D represent the motor with no nucleotide bound and the motor with ADP bound (the same as those explained above), while in state $\mathrm{T}$ the motor carries ATP, ADP and $\mathrm{P}_{\mathrm{i}}$, or an intermediate object that appears in the course of the ATP hydrolysis, Eq. (1). In the present model it is assumed, as explained in detail later in this subsection, that the ATP hydrolysis of Eq. (1) proceeds as the motor moves along the MT toward the direction of increasing $x$ (the reverse reaction, the ATP synthesis, occurs if the motor moves in the opposite direction) in state T. In this three-state model, a transition from one state to another takes place upon the binding or unbinding of a nucleotide as indicated in Fig. 2.

Concerning the potentials representing the interaction between the motor and the track, we adopt those used in the two-state ratchet model. The potentials in states $\Phi$ and T are given by

$$
V_{\Phi}(x)=V_{0}\left(x-x_{\mathrm{T}}\right)
$$

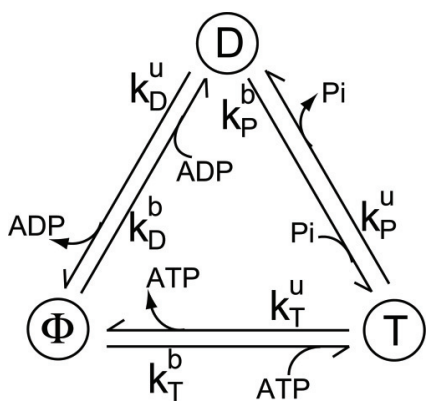

FIG. 2. Three states $\Phi, \mathrm{T}$, and D considered in the present model, and the transitions between pairs of states. Symbols $k_{\alpha}^{\mathrm{b}}$ and $k_{\alpha}^{\mathrm{u}}(\alpha=$ $\mathrm{T}, \mathrm{P}, \mathrm{D})$ are rate constants associated with the biding and unbinding, respectively, of nucleotide $\alpha$, which trigger the transitions between the states. In state T the ATP hydrolysis [Eq. (1)] or its reverse reaction (ATP synthesis) occurs as the motor moves forward (increasing $x$ ) or backward along the MT. 
and

$$
V_{\mathrm{T}}(x)=V_{0}\left(x-x_{\mathrm{P}}\right),
$$

respectively, where $V_{0}$ is the sawtooth potential defined in Eq. (2), $x$ is the location of the motor, and $x_{\mathrm{T}}$ and $x_{\mathrm{P}}$ are certain constants (the positions of minima of $V_{\Phi}$ and $V_{\mathrm{T}}$ ); remember that these states are strong-binding states of KIF1A. In state $\mathrm{D}$, which is the weak-binding state, the potential is assumed to be given by

$$
V_{\mathrm{D}}(x)=\text { const. }
$$

It is plausible that the conformation (structure) of the motor protein changes as the motor moves along the track: the interaction between the motor and track will cause a conformational change in a region of the motor in contact with the track, and this change may affect the conformation of another region (allosteric interaction [1]). Possible conformational changes in the nucleotide-binding site associated with the translational motion of the motor can control the binding and unbinding of nucleotides and the catalytic reaction of ATP hydrolysis and synthesis. We shall assume that this is the case and that each value of $x$ corresponds to a certain conformation of the motor. In this regard we should think of potential $V_{\Phi}(x)$ as representing not only the interaction between the motor and the track but also the interactions between atoms (ions) constituting the motor. Note that $V_{\mathrm{T}}(x)$ and $V_{\mathrm{D}}(x)$ include the additional interactions arising from the nucleotides bound to the motor.

In order to simplify the analysis, we assume that the binding and unbinding of nucleotides can occur only if the motor is at particular positions on the MT (which corresponds to particular conformations of the motor). Let $x_{\alpha}(\bmod L)$ be the location at which the motor can bind or unbind nucleotide $\alpha(\alpha=$ $\mathrm{T}, \mathrm{D}, \mathrm{P})$ and assume that the following relation is satisfied (see Fig. 3):

$$
0 \leqslant x_{\mathrm{T}}<x_{\mathrm{P}}<x_{\mathrm{D}}<L,
$$

where $x_{\mathrm{T}}$ and $x_{\mathrm{P}}$ are the same as those in Eqs. (4) and (5). For later use, we introduce two distances,

$$
\delta_{1}=\left(x_{\mathrm{T}}+L\right)-x_{\mathrm{D}}, \quad \delta_{2}=x_{\mathrm{P}}-x_{\mathrm{T}} .
$$

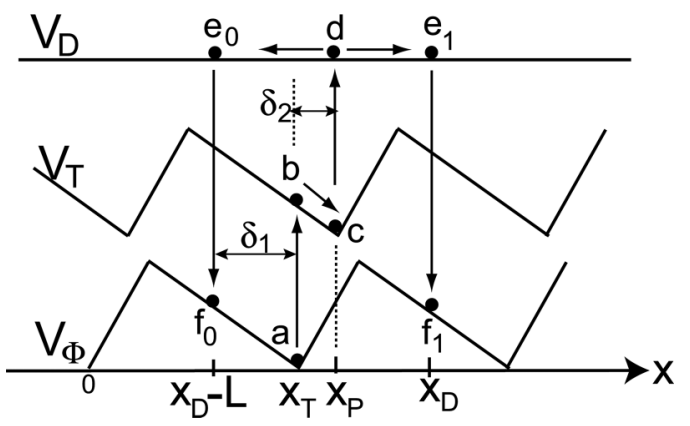

FIG. 3. The potentials in states D, T, and $\Phi$ in the present model and the typical transition path ways starting from the location labeled a in state $\Phi$. The graphs of $V_{\mathrm{T}}$ and $V_{\mathrm{D}}$ are vertically shifted for clarity. Vertical arrows $\mathrm{a} \rightarrow \mathrm{b}, \mathrm{c} \rightarrow \mathrm{d}$, and $\mathrm{e}_{n} \rightarrow \mathrm{f}_{n}(n=0, \pm 1 \ldots)$ indicate transitions $\Phi \rightarrow \mathrm{T}, \mathrm{T} \rightarrow \mathrm{D}$, and $\mathrm{D} \rightarrow \Phi$, respectively, in Fig. 2. The ATP hydrolysis reaction (1) proceeds as the motor moves from $\mathrm{b}$ to $\mathrm{c}$.
Right after an ATP molecule binds to the motor, it is located at $x=x_{\mathrm{T}}$ on the track and it is in state $\mathrm{T}$. We will assume that the reaction (1), the ATP hydrolysis, proceeds as $x$ increases from $x_{\mathrm{T}}$ and completes before it reaches $x_{\mathrm{P}}$ if the motor is in state T. Conversely, the reverse reaction (ATP synthesis) proceeds as $x$ decreases from $x_{\mathrm{P}}$ and completes before it reaches $x_{\mathrm{T}}$ in state $\mathrm{T}$.

It would be instructive to provide a qualitative explanation of how the motor may work in the present model before we go into the detailed analysis. Suppose that the chemical energy $\Delta \mu$ released in a cycle of the hydrolysis reaction is much larger than the thermal energy and the external force is absent. Then the ATP hydrolysis reaction, i.e., the counterclockwise flow of transitions $(\Phi \rightarrow \mathrm{T} \rightarrow \mathrm{D} \rightarrow \Phi)$ in Fig. 2, is dominant against the ATP synthesis (the clockwise flow of transitions in Fig. 2). Let us start with state $\Phi$, and the motor is supposed to sit at $x=$ $x_{\mathrm{T}}$; see point a in Fig. 3. When an ATP molecule is bound to the motor, a transition $\Phi \rightarrow \mathrm{T}$ occurs at the same location (arrow $\mathrm{a} \rightarrow \mathrm{b}$ in Fig. 3). Then the motor slides down the slope of potential $V_{\mathrm{T}}$ to point $\mathrm{c}$ in Fig. 3 at $x=x_{\mathrm{P}}$, resulting in the displacement of $\delta_{2}$; the hydrolysis reaction (1) takes place in this process as we assumed above. The release of a $\mathrm{P}_{\mathrm{i}}$ molecule at point $\mathrm{c}$ causes the transition to sate $\mathrm{D}$ (arrow $\mathrm{c} \rightarrow \mathrm{d}$ in Fig. 3), and the diffusion of the motor follows. After a while, a transition to state $\Phi$ associated with the release of an ADP molecule occurs at one of locations

$$
x_{n}=x_{\mathrm{D}}+(n-1) L \quad(n=0, \pm 1, \pm 2, \ldots),
$$

which are marked $\mathrm{e}_{0}, \mathrm{e}_{ \pm 1}, \ldots$ in Fig. 3. It is not difficult to see that the statistical average of the displacement $x_{n}-x_{\mathrm{P}}$ of the motor in state D is zero (see Appendix A 1). Immediately after the transition to state $\Phi$, the motor slides down the slope of potential $V_{\Phi}$ by distance $\delta_{1}$ to a minimum of $V_{\Phi}$; this distance may correspond to the displacement of $2.8 \pm 0.8 \mathrm{~nm}$ observed experimentally upon the binding of KIF1A to the MT [13] (see Appendix A2). Thus, the motor moves by distance

$$
\delta=\delta_{1}+\delta_{2}
$$

on average toward the plus direction of the $x$ axis in a cycle of transitions $\Phi \rightarrow \mathrm{T} \rightarrow \mathrm{D} \rightarrow \Phi$. Okada et al. [13] estimated the value of $\delta$ to be $3.6 \pm 1 \mathrm{~nm}$ experimentally.

How is the chemical energy $\Delta \mu$ given in Eq. (3) consumed in the cycle described above? First, it should be noted that the free energy of the environment (the reservoir of the nucleotides) decreases by $\Delta \mu$ in the whole cycle and not in the single event of the ATP hydrolysis $(b \rightarrow c$ in Fig. 3) that proceeds in state $\mathrm{T}$ : The free energy changes by $-\mu_{\mathrm{T}}$, $\mu_{\mathrm{P}}$, and $\mu_{\mathrm{D}}$ upon transitions $\Phi \rightarrow \mathrm{T}, \mathrm{T} \rightarrow \mathrm{D}$, and $\mathrm{D} \rightarrow \Phi$, respectively. Certain fractions of these free-energy changes are used to raise the potential energies of the motor in states $\mathrm{T}$ and $\Phi$, and the rest is dissipated as heat. The raised potential energies are used to push the motor by sliding down the potential slopes ( $\mathrm{b} \rightarrow \mathrm{c}$ and $\mathrm{f} \rightarrow \mathrm{a}$ in Fig. 3), and these energies will be dissipated as heat eventually; if the external load force is present (as discussed later) part of these energies is used as mechanical work against the load. 


\section{Fokker-Planck equation}

In the qualitative explanation given above, the effects of thermal fluctuations in the strong-binding states (states $\Phi$ and $\mathrm{T}$ ) are neglected. However, the dynamics of the translational motion of the motor in any state should be described by the following overdamped Langevin equation:

$$
\gamma \frac{d x}{d t}=-\frac{d V_{i}(x)}{d x}-F+\xi(t) \quad(i=\Phi, \mathrm{T}, \mathrm{D}),
$$

where $\gamma$ is the coefficient of viscous drag, $V_{i}$ is the potential in state $i, F$ is a constant external force (load), and $\xi$ is the Gaussian white noise which satisfies the following fluctuation dissipation theorem:

$$
\langle\xi(t)\rangle=0, \quad\left\langle\xi(t) \xi\left(t^{\prime}\right)\right\rangle=2 \gamma k_{\mathrm{B}} T \delta\left(t-t^{\prime}\right),
$$

where $\langle\cdots\rangle$ indicates the statistical average, $k_{\mathrm{B}}$ is the Boltzmann constant, and $T$ is the temperature. We have included the load force in Eq. (11) so the mechanical work and the power output delivered by the motor can be investigated.

In Eq. (11), potential $V_{i}$ is switched to $V_{j}$ if the transition from state $i$ to state $j$ takes place. Since a transition is associated with binding or unbinding of a nucleotide at particular locations $\left(x_{\mathrm{T}}, x_{\mathrm{P}}\right.$, or $\left.x_{\mathrm{D}}\right)$ of the motor, the rate $\omega_{i j}(x)$ of transition from state $j$ to state $i$ at location $x$ can be expressed as

$$
\omega_{i j}(x)=k_{i j} \sum_{n=-\infty}^{\infty} \delta\left(x-x_{i j}+n L\right),
$$

where $x_{\mathrm{T} \Phi}$ and $x_{\Phi \mathrm{T}}$ are identical to $x_{\mathrm{T}}, x_{\mathrm{TD}}$ and $x_{\mathrm{DT}}$ to $x_{\mathrm{P}}$, and $x_{\mathrm{D} \Phi}$ and $x_{\Phi \mathrm{D}}$ to $x_{\mathrm{D}}$; the rate constant $k_{i j}$ is identified with a binding rate $k_{\alpha}^{\mathrm{b}}$ or an unbinding rate $k_{\alpha}^{\mathrm{u}}$ of nucleotide $\alpha(\alpha=\mathrm{T}$, $\mathrm{D}, \mathrm{P})$ :

$$
\begin{array}{ll}
k_{\mathrm{T} \Phi}=k_{\mathrm{T}}^{\mathrm{b}}, & k_{\Phi \mathrm{T}}=k_{\mathrm{T}}^{\mathrm{u}}, \quad k_{\mathrm{TD}}=k_{\mathrm{P}}^{\mathrm{b}}, \quad k_{\mathrm{DT}}=k_{\mathrm{P}}^{\mathrm{u}}, \\
k_{\mathrm{D} \Phi}=k_{\mathrm{D}}^{\mathrm{b}}, & k_{\Phi \mathrm{D}}=k_{\mathrm{D}}^{\mathrm{u}} .
\end{array}
$$

Note that $k_{i j}$ has dimensions of (time $)^{-1} \times$ (length) not (time $)^{-1}$ because of the $\delta$ function in Eq. (13). These rate constants are indicated also in Fig. 2. The use of the $\delta$ function in Eq. (13) is a mathematical idealization, which has been introduced by several authors $[8,15,18-22]$ to carry out various calculations analytically.

From the detailed balance condition, the following relations between the rate constants $k_{\alpha}^{\mathrm{b}}$ and $k_{\alpha}^{\mathrm{u}}$ are derived:

$$
\frac{k_{\alpha}^{\mathrm{b}}}{k_{\alpha}^{\mathrm{u}}}=\exp \left[\beta\left(\mu_{\alpha}-\Delta V_{\alpha}\right)\right] \quad(\alpha=\mathrm{T}, \mathrm{P}, \mathrm{D}),
$$

where $\beta=1 / k_{\mathrm{B}} T$ and $\Delta V_{\alpha}$ are defined by

$$
\begin{gathered}
\Delta V_{\mathrm{T}}=V_{\mathrm{T}}\left(x_{\mathrm{T}}\right)-V_{\Phi}\left(x_{\mathrm{T}}\right), \\
\Delta V_{\mathrm{P}}=V_{\mathrm{T}}\left(x_{\mathrm{P}}\right)-V_{\mathrm{D}}\left(x_{\mathrm{P}}\right), \\
\Delta V_{\mathrm{D}}=V_{\mathrm{D}}\left(x_{\mathrm{D}}\right)-V_{\Phi}\left(x_{\mathrm{D}}\right) .
\end{gathered}
$$

Noting that the right-hand side in Eq. (15) is proportional to the concentration of nucleotide $\alpha$ in solution, we define a "reduced concentration" $\rho_{\alpha}$ of nucleotide $\alpha$ by

$$
\rho_{\alpha}=\exp \left[\beta\left(\mu_{\alpha}-\Delta V_{\alpha}\right)\right] \quad(\alpha=\mathrm{T}, \mathrm{P}, \mathrm{D}) .
$$

In the present work, the binding rate of nucleotide will be assumed to be proportional to its concentration; this implies that the dissociation (unbinding) rate is independent of the concentration due to Eq. (15). Hence, we have

$$
k_{\alpha}^{\mathrm{b}}=\kappa_{\alpha} \rho_{\alpha}, \quad k_{\alpha}^{\mathrm{u}}=\kappa_{\alpha} \quad(\alpha=\mathrm{T}, \mathrm{P}, \mathrm{D})
$$

from Eqs. (15) and (19) with some positive constant $\kappa_{\alpha}$.

It is more convenient to work with the Fokker-Planck equation,

$\frac{\partial P_{i}}{\partial t}+\frac{\partial J_{i}}{\partial x}=\sum_{j=\Phi, \mathrm{T}, \mathrm{D}}\left[-\omega_{j i} P_{i}+\omega_{i j} P_{j}\right] \quad(i=\Phi, \mathrm{T}, \mathrm{D})$,

for probability densities $P_{i}(x, t)$ of the motor position in state $i$, instead of the Langevin equation (11). In Eq. (21),

$$
J_{i}(x, t)=-D_{0}\left(\frac{\partial}{\partial x}+\frac{d U_{i}}{d x}\right) P_{i} \quad(i=\Phi, \mathrm{T}, \mathrm{D})
$$

is the probability current in state $i$, where $U_{i}(x)$ is the "effective potential" defined by

$$
U_{i}(x)=\beta\left[V_{i}(x)+F x\right] .
$$

In Eq. (22), $D_{0}=k_{\mathrm{B}} T / \gamma$ is the diffusion coefficient of the motor. The diffusion coefficient of KIF1A reported in Ref. [13] is $26 \pm 6 \mathrm{~nm}^{2} / \mathrm{ms}$ at $T=299 \pm 1 \mathrm{~K}$.

\section{Steady state}

We are interested in the steady state $\left[\partial P_{i}(x, t) / \partial t=0\right]$ of the model. Let $P_{i}(x)$ be the steady-state solution of the FokkerPlanck equation (21). We impose the periodic condition

$$
P_{i}(x+L)=P_{i}(x)
$$

and the normalization condition

$$
\int_{0}^{L}\left[P_{\Phi}(x)+P_{\mathrm{T}}(x)+P_{\mathrm{D}}(x)\right] d x=1
$$

on $P_{i}(x)$. In the steady state, the total probability current

$$
J \equiv J_{\Phi}(x)+J_{\mathrm{T}}(x)+J_{\mathrm{D}}(x)
$$

is independent of $x$ and is related with the average velocity $v$ of the motor as

$$
v=J L .
$$

The average reaction rate (consumption of ATP molecules per unit time) $r$ is given by any of the following three expressions:

$$
\begin{aligned}
r & =k_{\mathrm{T}}^{\mathrm{b}} P_{\Phi}\left(x_{\mathrm{T}}\right)-k_{\mathrm{T}}^{\mathrm{u}} P_{\mathrm{T}}\left(x_{\mathrm{T}}\right)=k_{\mathrm{P}}^{\mathrm{u}} P_{\mathrm{T}}\left(x_{\mathrm{P}}\right)-k_{\mathrm{P}}^{\mathrm{b}} P_{\mathrm{D}}\left(x_{\mathrm{P}}\right) \\
& =k_{\mathrm{D}}^{\mathrm{u}} P_{\mathrm{D}}\left(x_{\mathrm{D}}\right)-k_{\mathrm{D}}^{\mathrm{b}} P_{\Phi}\left(x_{\mathrm{D}}\right) .
\end{aligned}
$$

It is apparent from the Fokker-Planck equation (21) and the periodicity (24) of $P_{i}(x)$ that these expressions yield the same result.

The Fokker-Planck equation (21) with piecewise linear potentials $U_{i}$ and transition rates $\omega_{i j}(x)$ given as sums of the $\delta$ function can be solved analytically for the steady-state solution $P_{i}(x)$, as explained in Appendix B. From this solution, the average velocity $v$ and the reaction rate $r$ are obtained by using Eqs. (27) and (28), respectively. The results may be expressed as the quotient of multilinear functions of $\rho_{\mathrm{T}}, \rho_{\mathrm{D}}$, and $\rho_{\mathrm{P}}$ :

$$
r=\frac{Z_{r}}{R}, \quad v=\frac{Z_{v} L}{R},
$$


where $R, Z_{r}$, and $Z_{v}$ are given by the following equations:

$$
\begin{gathered}
R=\sum_{l=0}^{1} \sum_{m=0}^{1} \sum_{n=0}^{1} C_{l m n} \rho_{\mathrm{T}}^{l} \rho_{\mathrm{D}}^{m} \rho_{\mathrm{P}}^{n}, \\
Z_{r}=\rho_{\mathrm{T}}-\sigma \rho_{\mathrm{D}} \rho_{\mathrm{P}}, \\
Z_{v}=\sum_{l=0}^{1} \sum_{m=0}^{1} \sum_{n=0}^{1} \lambda_{l m n} \rho_{\mathrm{T}}^{l} \rho_{\mathrm{D}}^{m} \rho_{\mathrm{P}}^{n} .
\end{gathered}
$$

The coefficients $C_{l m n}, \sigma$, and $\lambda_{l m n}$ in these equations are given in Eqs. (B23)-(B39) in Appendix B. They depend on the rate constants $\kappa_{\alpha}$, the diffusion coefficient $D_{0}$, the shapes of potentials $V_{i}(x)$, and the load force $F$. Coefficients $C$ 's have dimensions of time, while $\sigma$ and $\lambda$ 's are dimensionless.

It is noted that $R$ is positive because all $C$ 's are positive. Therefore, the signs of $r$ and $v$ are determined by the signs of $Z_{r}$ and $Z_{v}$, respectively. In particular, in the case of no external load $(F=0), Z_{r}$ is given by

$$
Z_{r}=\rho_{\mathrm{T}}[1-\exp (-\beta \Delta \mu)],
$$

as shown in Appendix $\mathrm{C} 1$. Here $\Delta \mu$ defined in Eq. (3) is the free energy released by the hydrolysis of a single ATP molecule and is given by

$$
\Delta \mu=k_{\mathrm{B}} T \ln \frac{\rho_{\mathrm{T}}}{\rho_{\mathrm{D}} \rho_{\mathrm{P}}}+\frac{W \delta}{L-a}
$$

in the present model. This relation comes from Eqs. (3) and (19); note that the second term of the right side in Eq. (34) corresponds to $\Delta V_{\mathrm{T}}-\Delta V_{\mathrm{D}}-\Delta V_{\mathrm{P}}$. We see from Eq. (33) that $r>0$ for $\Delta \mu>0$, which may be expected from the second law of thermodynamics.

\section{REACTION RATE AND MODEL PARAMETERS}

\section{A. Approximate expression for $\boldsymbol{r}$ without load}

The exact results for the reaction rate $r$ and the velocity $v$ obtained in the preceding section will be used to calculate various properties in the following sections. However, it is difficult to gain physical insight from the complicated expressions for $r$ and $v$ given by Eq. (29) with Eqs. (30)-(32) and Eqs. (B23)-(B40). In this section, we present a useful approximate expression for $r$, which is valid under most experimental conditions for KIF1A. From this expression and certain experimental results for KIF1A, we will determine the parameter values in the present model.

Let us consider the case of no external load $(F=0)$ and assume that conditions

$$
e^{\beta W} \gg 1, \quad e^{\beta \Delta \mu} \gg 1
$$

are satisfied. Then, as shown in Appendix C 1, the dependence of the reaction rate on the reduced ATP concentration $\rho_{\mathrm{T}}$ can be approximated by a Michaelis-Menten-type equation,

$$
r \simeq \frac{r_{\max } \rho_{\mathrm{T}}}{K_{\mathrm{m}}+\rho_{\mathrm{T}}},
$$

where the saturating rate $r_{\max }$ and the Michaelis constant $K_{\mathrm{m}}$ depend on the reduced ADP concentration $\rho_{\mathrm{D}}$ as follows:

$$
\begin{aligned}
r_{\text {max }} & \simeq \frac{1}{C_{\mathrm{T}}+C_{\mathrm{TD}} \rho_{\mathrm{D}}}, \\
K_{\mathrm{m}} & \simeq \frac{C_{0}+C_{\mathrm{D}} \rho_{\mathrm{D}}}{C_{\mathrm{T}}+C_{\mathrm{TD}} \rho_{\mathrm{D}}} .
\end{aligned}
$$

Here, the coefficients $C_{0}, C_{\mathrm{T}}, C_{\mathrm{D}}$, and $C_{\mathrm{TD}}$ are identical to $C_{000}, C_{100}, C_{010}$, and $C_{110}$ in Eq. (30), respectively, and they are approximately given by

$$
\begin{gathered}
C_{0} \simeq \frac{L}{\beta W}\left[\frac{(L-a)\left(1-\varepsilon_{2}\right)}{\beta W D_{0}}+\frac{1}{\kappa_{\mathrm{T}}}+\frac{\varepsilon_{2}}{\kappa_{\mathrm{P}}}\right], \\
C_{\mathrm{T}} \simeq \tau_{\mathrm{D}}+\tau_{\Phi}+\tau_{\mathrm{T}}, \\
C_{\mathrm{D}} \simeq \beta W \varepsilon_{1} C_{0}, \\
C_{\mathrm{TD}} \simeq \frac{L(L-a)}{\beta W D_{0}}\left(1-\varepsilon_{1}\right),
\end{gathered}
$$

where $\varepsilon_{i}(i=1,2)$ are defined by

$$
\varepsilon_{i}=\exp \left(-\frac{\beta W \delta_{i}}{L-a}\right)
$$

and $\tau$ 's in Eq. (40) by

$$
\begin{gathered}
\tau_{\mathrm{D}}=\frac{(L-\delta) \delta}{2 D_{0}}+\frac{L}{\kappa_{\mathrm{D}}}, \\
\tau_{\Phi}=\frac{(L-a) \delta_{1}}{\beta W D_{0}}, \\
\tau_{\mathrm{T}}=\frac{(L-a) \delta_{2}}{\beta W D_{0}}+\frac{L}{\beta W \kappa_{\mathrm{P}}} .
\end{gathered}
$$

It is noted that the reduced concentration $\rho_{\mathrm{P}}$ of $\mathrm{P}_{\mathrm{i}}$ does not appear in any of approximate expressions given above. This is mainly because $\rho_{\mathrm{P}}$ is extremely small (less than $\sim 10^{-5}$ ) for reasonable concentrations of $\mathrm{P}_{\mathrm{i}}\left(\left[\mathrm{P}_{\mathrm{i}}\right]<100 \mathrm{mM}\right)$, as explained in the next subsection.

In the limit of large $\rho_{\mathrm{T}}$ and small $\rho_{\mathrm{D}}$, we obtain

$$
r=1 /\left(\tau_{\mathrm{D}}+\tau_{\Phi}+\tau_{\mathrm{T}}\right)
$$

from Eqs. (36), (37), and (40). The contributions $\tau_{\mathrm{D}}, \tau_{\Phi}$, and $\tau_{\mathrm{T}}$ to the denominator in Eq. (47) can be interpreted as the lifetimes of states $\mathrm{D}, \Phi$, and T, respectively, in this limit for the following reasons. In Eq. (44), the first term is roughly the time needed for the motor to diffuse from point $\mathrm{d}$ in Fig. 3 to points $\mathrm{e}_{0}$ or $\mathrm{e}_{1}$, and the second term represents the average waiting time for the ADP release (see Appendix A 3). The right-hand side of Eq. (45) is the average time needed for the motor to slide down the slope of potential $V_{\Phi}(x)$ by distance $\delta_{1}$ from point $\mathrm{f}_{0}$ to point a in Fig. 3; note that the waiting time for the motor to bind an ATP molecule is negligibly small in the limit of large $\rho_{\mathrm{T}}$. In Eq. (46), the first term is the time needed for the motor to slide down the slope of potential $V_{\mathrm{T}}(x)$ by distance $\delta_{2}$ from point $\mathrm{b}$ to point $\mathrm{c}$ in Fig. 3, and the second term represents the average waiting time for the $\mathrm{P}_{\mathrm{i}}$ release.

\section{B. Parameter values}

As explained in Sec. II, we have $L=8 \mathrm{~nm}$ from the periodicity of the microtubule and have set $\delta_{1}=2.8 \mathrm{~nm}$, $\delta_{2}=0.8 \mathrm{~nm}, D_{0}=2.6 \times 10^{4} \mathrm{~nm}^{2} / \mathrm{s}$, and $T=300 \mathrm{~K}$ from the consideration of the experiment by Okada et al. [13]. Concerning the height $W$ of potentials $V_{\Phi}$ and $V_{\mathrm{T}}$ and parameter $a$ associated with the asymmetry of these potentials, we do not have direct experimental information. In the present model, as long as $W$ is much larger than the thermal energy $k_{\mathrm{B}} T$, the results does not change qualitatively with $W$ and $a$. 
We use $W=12 k_{\mathrm{B}} T$ and $a=1.6 \mathrm{~nm}$ in this work; the latter value was used in Ref. [17].

The rate constants $\kappa_{\mathrm{D}}$ and $\kappa_{\mathrm{P}}$ can be estimated by using the expressions given in the preceding subsection. The lifetime $\tau_{\mathrm{D}}$ of state D was measured by Okada et al. [13] to be $4 \pm 1 \mathrm{~ms}$; from this value and Eq. (44) we have $\kappa_{\mathrm{D}} \sim 2 \times 10^{3} \mathrm{~nm} / \mathrm{s}$. Then the information on the reaction rate in the limit of large $\rho_{\mathrm{T}}$ and small $\rho_{\mathrm{D}}$, Eq. (47), can be used to estimate $\kappa_{\mathrm{P}}$ with the aid of Eqs. (45) and (46). This rate in solution was measured by Okada et al. [13] to be $108 \pm 6 \mathrm{~s}^{-1}$; however, in the motility experiment with optical trap by the same authors, this rate seems to be about $60 \mathrm{~s}^{-1}$ [23]. We adopt the latter value (since we are interested in the motility of KIF1A under the external load) and have $\kappa_{\mathrm{P}} \sim 50 \mathrm{~nm} / \mathrm{s}$. We shall use $\kappa_{\mathrm{D}}=2.2 \times 10^{3} \mathrm{~nm} / \mathrm{s}$ and $\kappa_{\mathrm{P}}=47 \mathrm{~nm} / \mathrm{s}$.

We are not aware of experimental data on KIF1A that can be used to determine the rate constant $\kappa_{\mathrm{T}}$. We consult the data on a monomeric construct of conventional kinesin, a close cousin of KIF1A in the kinesin superfamily, reported in Ref. [24]: The rate of ATP release is $200 \pm 67 \mathrm{~s}^{-1}$. We tentatively identify this rate with $\kappa_{\mathrm{T}} / \delta_{2}$ in our model, and adopt $\kappa_{\mathrm{T}}=1.6 \times 10^{2} \mathrm{~nm} / \mathrm{s}$. We have checked that the results presented in the following sections are insensitive to the value of $\kappa_{\mathrm{T}}$ as long as it lies in the range from $10^{-3}$ to $5 \times 10^{3} \mathrm{~nm} / \mathrm{s}$.

In order to compare the theoretical results with the experiment and to make some predictions, we need to clarify the relations between the reduced concentrations $\rho_{\alpha}$ of nucleotides and actual concentrations $[\mathrm{ATP}],[\mathrm{ADP}]$, and $\left[\mathrm{P}_{\mathrm{i}}\right]$, i.e., the proportionality constants $A$ in the relations

$$
[\mathrm{ATP}]=A_{\mathrm{T}} \rho_{\mathrm{T}}, \quad[\mathrm{ADP}]=A_{\mathrm{D}} \rho_{\mathrm{D}}, \quad\left[\mathrm{P}_{\mathrm{i}}\right]=\mathrm{A}_{\mathrm{P}} \rho_{\mathrm{P}}
$$

should be given. Constant $A_{\mathrm{T}}$ can be determined by considering the Michaelis constant $K_{\mathrm{m}}$ given in Eq. (38). The Michaelis constant of KIF1A in the absence of ADP was found to be $63 \pm 10 \mu \mathrm{M}$ [13], which should be identified with $\left(C_{0} / C_{\mathrm{T}}\right) A_{\mathrm{T}}$. Since $C_{0}$ and $C_{\mathrm{T}}$ can be estimated $\left(C_{0}=7.34 \mathrm{~ms}\right.$ and $C_{\mathrm{T}}=$ $18.2 \mathrm{~ms}$ ) from Eqs. (39) and (40) with already determined parameters, we obtain $A_{\mathrm{T}} \sim 1.6 \times 10^{2} \mu \mathrm{M}$. Now $A_{\mathrm{D}}$ may be determined from the dependence of the reaction rate on [ADP] with the saturating concentration of [ATP] measured by Okada et al. [12]. This dependence should be compared with $r_{\max }$ given in Eq. (37), which predicts that the rate decreases with increasing $\rho_{\mathrm{D}}$ and becomes halved at $\rho_{\mathrm{D}}=C_{\mathrm{T}} / C_{\mathrm{TD}}$ compared to the rate at $\rho_{\mathrm{D}}=0$. This behavior is consistent with what is reported in Ref. [12], where the rate becomes halved at $[\mathrm{ADP}] \sim 1.2 \mathrm{mM}$; this value corresponds to $\left(C_{\mathrm{T}} / C_{\mathrm{TD}}\right) A_{\mathrm{D}}$ in our model, and we obtain $A_{\mathrm{D}} \sim 1.1 \times 10^{-2} \mathrm{mM}$. Once $A_{\mathrm{T}}$ and $A_{\mathrm{D}}$ are determined, $A_{\mathrm{P}}$ may be obtained from the well-known expression

$$
\Delta \mu=\Delta \mu_{0}+k_{\mathrm{B}} T \ln \left([\mathrm{ATP}] /[\mathrm{ADP}]\left[\mathrm{P}_{\mathrm{i}}\right]\right)
$$

for the fee energy change due to the hydrolysis of one ATP molecule, where $\Delta \mu_{0}=54 \times 10^{-21} \mathrm{~J}$ with the convention that the concentrations of the nucleotides be expressed in $\mathrm{M}$ (molar) [2]. Substituting Eq. (48) into Eq. (49) and comparing the latter with Eq. (34), we obtain $A_{\mathrm{P}} \sim 8 \times 10^{6} \mathrm{mM}$. From these considerations, we adopt $A_{\mathrm{T}}=0.15 \mathrm{mM}, A_{\mathrm{D}}=1.1 \times$ $10^{-2} \mathrm{mM}$, and $A_{\mathrm{P}}=8.1 \times 10^{6} \mathrm{mM}$. It is remarked that $A_{\mathrm{P}}$ is several orders of magnitude larger than $A_{\mathrm{T}}$ and $A_{\mathrm{D}}$. Due to this fact, the dimensionless concentration $\rho_{\mathrm{P}}$ of $\mathrm{P}_{\mathrm{i}}$ is extremely

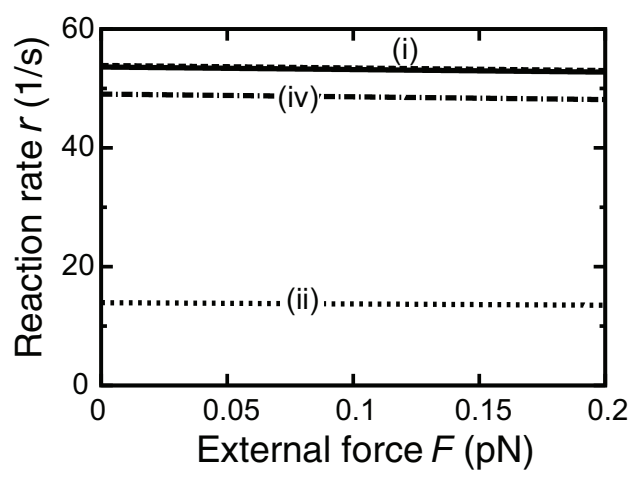

FIG. 4. The dependence of reaction rate $r$ on load $F$ for different concentrations of ATP and ADP while the phosphate concentration is fixed as $\left[\mathrm{P}_{\mathrm{i}}\right]=1.6 \mathrm{mM}$. The condition ([ATP], $\left.[\mathrm{ADP}]\right)=$ $(2.0 \mathrm{mM}, 5.0 \mu \mathrm{M})$ for the solid line is close to the physiological one. The conditions for the other lines are (i) ([ATP], [ADP]) = (2.0 mM,0.1 $\mu \mathrm{M})$, (ii) $(21 \mu \mathrm{M}, 0.1 \mu \mathrm{M})$, and (iv) $(2.0 \mathrm{mM}, 100 \mu \mathrm{M})$. The roman numbers i, ii, and iv correspond to those used in Fig. 6.

small $\left(\rho_{\mathrm{P}}<1.1 \times 10^{-5}\right.$ for $\left.\left[\mathrm{P}_{\mathrm{i}}\right]<100 \mathrm{mM}\right)$, as mentioned in the preceding subsection.

In the present paper, we shall assume, unless otherwise stated, that the concentrations of the nucleotides are in the ranges

$[\mathrm{ATP}] \geqslant 10^{-4} \mathrm{mM}, \quad[\mathrm{ADP}] \leqslant 10^{2} \mathrm{mM}, \quad\left[\mathrm{P}_{\mathrm{i}}\right] \leqslant 10^{2} \mathrm{mM}$,

which cover the conditions in most of experiments.

\section{Dependence of $\boldsymbol{r}$ on $\boldsymbol{F}$}

In contrast to the simplicity of the dependence on the concentrations of nucleotides, the dependence of reaction rate $r$ on load $F$ is too complicated to work out analytically. The numerical results based on the exact expression for $r$ [Eq. (29)] are shown in Fig. 4, where we observe that $r$ is almost independent of $F$. Our numerical results with various choices of [ATP], [ADP], and $\left[\mathrm{P}_{\mathrm{i}}\right]$ other than those indicated in Fig. 4 show that $r$ is practically independent of $F$ if the conditions (35) and

$$
\beta F L \ll 1
$$

are satisfied. Since the second term in Eq. (31) is negligibly small compared to the first term under these conditions, it is concluded that $R$, the common denominator of the expressions for $r$ and $v$ in Eq. (29), is practically independent of $F$ in the same conditions.

\section{MOTILITY}

\section{A. The case of no load}

From the exact expressions (29) for velocity $v$ and reaction rate $r$, it can be shown (see Appendix $\mathrm{C} 2$ ) that the ratio $v / r$ is independent of [ATP], [ADP], and [ $\mathrm{P}_{\mathrm{i}}$ ] (i.e., $v$ and $r$ show the same dependence on the nucleotide concentrations) if the external load is absent $(F=0)$. If the conditions in Eq. (35) are also satisfied, the relation between $v$ and $r$ is simply given 
(a)

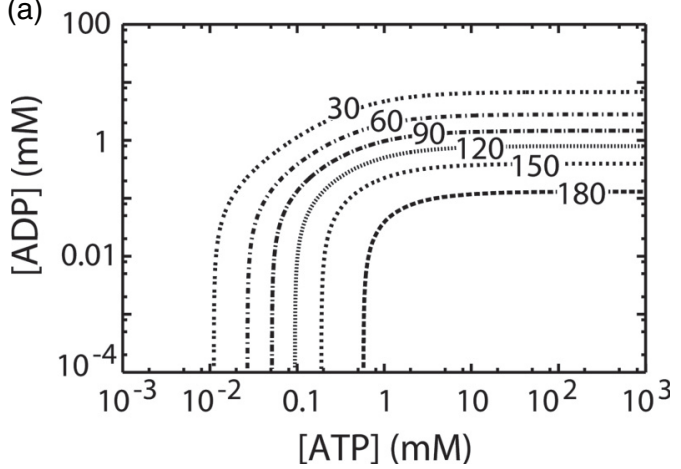

(b)

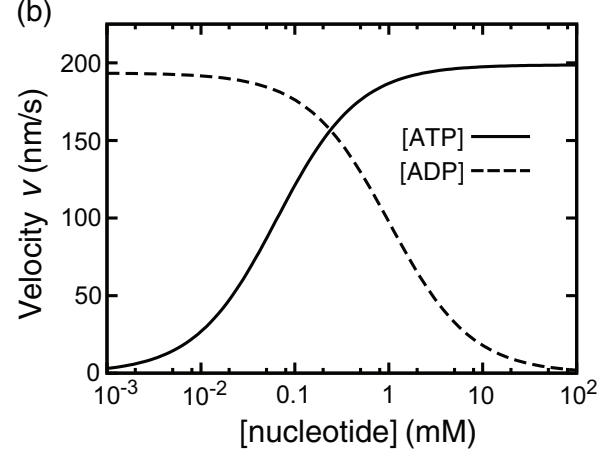

FIG. 5. Dependence of velocity $v$ on $[\mathrm{ATP}]$ and $[\mathrm{ADP}]$ for $F=0$ and $\left[\mathrm{P}_{\mathrm{i}}\right]=1.6 \mathrm{mM}$ obtained from Eq. (29). (a) The contour plot of $v$ on the ([ATP], [ADP]) plane, where the values of $v$ are indicated in units of $\mathrm{nm} / \mathrm{s}$. (b) The dependence of velocity $v$ on $[\mathrm{ATP}]$ for $[\mathrm{ADP}]=5.0 \mu \mathrm{M}$ (solid line) and on $[\mathrm{ADP}]$ for $[\mathrm{ATP}]=2.0 \mathrm{mM}$ (dotted line).

by

$$
v \simeq r \delta \quad(F=0),
$$

where $r$ is the reaction rate for $F=0$ given by Eq. (36). An intuitive explanation of Eq. (52) is as follows. Under the conditions considered here, the motor moves using mainly the pathways shown in Fig. 3 as discussed in Sec. II B, and, hence, the motor moves by distance $\delta$ in one cycle of ATP hydrolysis reaction (a counterclockwise cycle in Fig. 2), which implies the relation of Eq. (52).

Figure 5 shows the dependence of mean velocity $v$ on the nucleotide concentrations for $F=0$ based on the exact solution (29). The results shown in this figure can be explained by the approximate expression (52), which predicts that, as in the case of the reaction rate discussed in Sec. III A, the dependence of $v$ on [ATP] is the Michaelis-Menten type,

$$
v \simeq \frac{v_{\max } \rho_{\mathrm{T}}}{K_{\mathrm{m}}+\rho_{\mathrm{T}}}
$$

with $v_{\max }=r_{\max } \delta$, where $r_{\max }$ and $K_{\mathrm{m}}$ are given in Eqs. (37) and (38). An example of this dependence is shown in Fig. 5(b) as the solid line. This dependence of $v$ on [ATP] can be understood intuitively as follows. If the ATP concentration is low, the ATP binding process $\Phi \rightarrow \mathrm{T}(\mathrm{a} \rightarrow \mathrm{b}$ in Fig. 3) rarely occurs and the motor stays in state $\Phi$ for a while (i.e., the process $\Phi \rightarrow \mathrm{T}$ is late limiting). In this situation, the reaction rate $r$ can be approximated by the ATP binding rate, which is proportional to the ATP concentration, and, hence, the velocity $v \simeq r \delta$, Eq. (52), increases linearly with [ATP]. On the other hand, if [ATP] is large enough, the lifetime of state $\Phi$ becomes negligibly small and other processes, the rates of which are independent of [ATP], become rate limiting. Therefore, the velocity saturates to a certain value as $[\mathrm{ATP}]$ increases.

The dependence on [ADP] for large [ATP] can be well approximated by

$$
v \simeq \frac{\delta}{C_{\mathrm{T}}+C_{\mathrm{TD}} \rho_{\mathrm{D}}} \quad\left(\rho_{\mathrm{T}} \gg K_{\mathrm{m}}\right),
$$

which is obtained from Eqs. (36) and (52). An example of this dependence is shown in Fig. 5(b) as the dotted line. The velocity decreases as the ADP concentration is increased, because the ADP release process $\mathrm{D} \rightarrow \Phi$ becomes hindered by the increase in the rate of ADP binding $(\Phi \rightarrow \mathrm{D})$ and, hence, the reaction rate $r$ in Eq. (52) decreases.

The dependencies of $v$ on [ATP] for small [ADP] and on [ADP] for large [ATP] obtained here [the solid and dotted lines in Fig. 5(b)] are consistent with what were observed for KIF1A in Ref. [12]. We wish to see whether the dependence on [ATP] and [ADP] in wider ranges of the nucleotide concentrations predicted in Fig. 5(a) can be tested experimentally in the future.

\section{B. The case of small load}

Let us study the effect of external load $F$ on the velocity $v$. Figure 6(a) demonstrates the dependence of $v$ on $F$ calculated by using the exact expression (29). The velocity decreases almost linearly with increasing $F$ and becomes zero (the motor stalls) at a certain value of $F$, which is usually called a stall force. The dependence of the stall force $F_{\mathrm{s}}$ on [ATP] and [ADP] for $\left[\mathrm{P}_{\mathrm{i}}\right]=1.6 \mathrm{mM}$ is shown in Fig. $6(\mathrm{~b})$. Note that the stall force does not depend appreciably on [ATP] if [ATP] is not too small and [ADP] is small enough [see, for example, lines i, ii, and iii in Fig. 6(a)]. The linear dependence of $v$ on $F$ and the independence of $F_{\mathrm{s}}$ on [ATP] agree with the experimental results in Ref. [13]. However, we should emphasize that the stall force does depend on [ATP] and [ADP] if [ATP] is small or $[A D P]$ is large. It will not be difficult to observe this dependence experimentally.

As explained in Appendix C 3 , we are able to obtain a simple, approximate expression

$$
v \simeq\left(1-F / F_{\mathrm{s}}\right) r \delta,
$$

if $F$ is small enough and the conditions in Eq. (35) are satisfied. Here $r$ is the reaction rate for $F=0$ given by Eq. (36), and the stall force $F_{\mathrm{s}}$ is approximately given by

$$
F_{\mathrm{s}} \simeq \frac{\rho_{\mathrm{T}} \delta}{\beta D_{0}\left(C_{0}^{\prime}+\tau_{\mathrm{D}} \rho_{\mathrm{T}}+C_{\mathrm{D}} \rho_{\mathrm{D}}+C_{\mathrm{TD}} \rho_{\mathrm{T}} \rho_{\mathrm{D}}\right)},
$$

where

$$
C_{0}^{\prime}=(\beta W)^{2} e^{-\beta W} C_{0},
$$

and $C_{0}, C_{\mathrm{D}}, C_{\mathrm{TD}}$, and $\tau_{\mathrm{D}}$ have been defined in Eqs. (39), (41), (42), and (44), respectively. It turns out that Eqs. (55) and (56) 

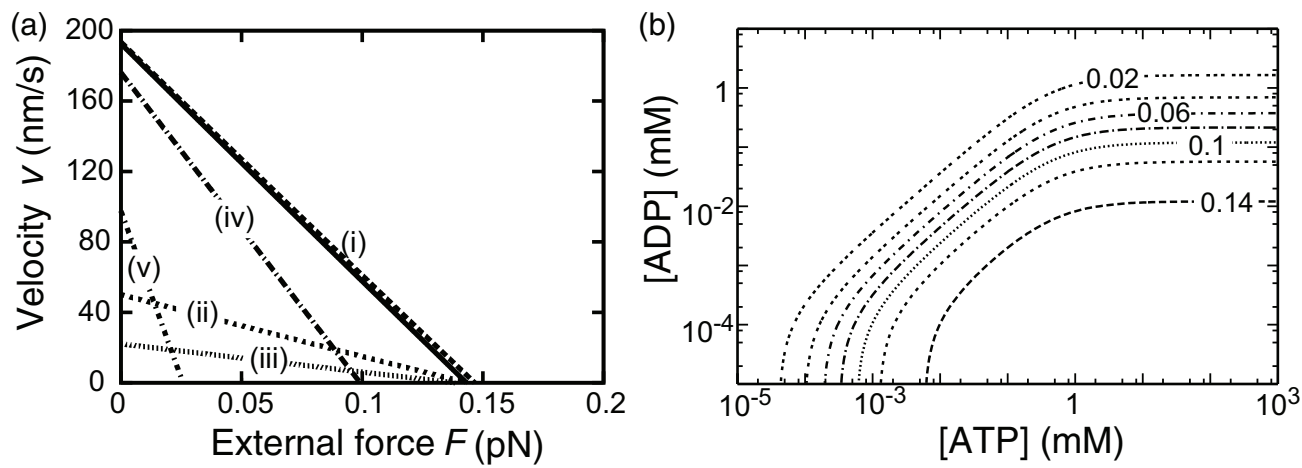

FIG. 6. (a) Dependence of velocity $v$ on load $F$ obtained from Eq. (29) for $\left[\mathrm{P}_{\mathrm{i}}\right]=1.6 \mathrm{mM}$ and different combinations of [ATP] and [ADP]. The condition $([\mathrm{ATP}],[\mathrm{ADP}])=(2.0 \mathrm{mM}, 5.0 \mu \mathrm{M})$ for the solid line is close to the physiological one. The conditions for the lines with the roman numbers are (i) $([\mathrm{ATP}],[\mathrm{ADP}])=(2.0 \mathrm{mM}, 0.1 \mu \mathrm{M})$, (ii) $(21 \mu \mathrm{M}, 0.1 \mu \mathrm{M})$, (iii) $(7.8 \mu \mathrm{M}, 0.1 \mu \mathrm{M})$, (iv) $(2.0 \mathrm{mM}, 100 \mu \mathrm{M})$, and (v) $(2.0 \mathrm{mM}, 1 \mathrm{mM})$. (b) The contour plot of stall force $F_{\mathrm{s}}$ on the ([ATP], [ADP]) plane for $\left[\mathrm{P}_{\mathrm{i}}\right]=1.6 \mathrm{mM}$, where the values of $F_{\mathrm{s}}$ are indicated in units of $\mathrm{pN}$.

explain the results shown in Fig. 6 semiquantitatively: for example, we have $F_{\mathrm{s}}=0.146$ and $0.102 \mathrm{pN}$ for the conditions corresponding to lines $\mathrm{i}$ and iv, respectively, by the above analytical expressions. The corresponding values are estimated as 0.147 and $0.102 \mathrm{pN}$ by solving the condition $v(F)=0$ with the exact equation (29) for $v$ : The relative errors in the approximate results are significantly small.

Expression (56) for the stall force is simplified as $F_{\mathrm{s}} \simeq$ $\delta / \beta D_{0} \tau_{\mathrm{D}}$, which is independent of nucleotide concentrations if $\rho_{\mathrm{T}} \gg 1$ and $\rho_{\mathrm{D}} \ll 1$. This simplified $F_{\mathrm{S}}$ together with the load dependence of $v$ given in Eq. (55) coincides with the ones derived in Ref. [13] based on a somewhat different model. It may be worth noting that the stall force in this extreme condition can be estimated by extending the qualitative argument given in Sec. II B as follows (see Appendix A 4 for the details). First, we note that processes $\mathrm{b} \rightarrow \mathrm{c}$ and $\mathrm{f}_{n} \rightarrow \mathrm{a}$ in Fig. 3 are practically unaffected by the load $F$ as small as a few tenths of a piconewton since the force of $W /(L-a) \simeq 7.7 \mathrm{pN}$ due to the potential slope is much larger than $F$. Therefore, the motor moves by distance $\delta$ in these processes; see Eq. (10). Second, the motor is pulled backward by the load force in the diffusion process in state $\mathrm{D}$, and it moves, on average, by a distance $\beta F D_{0} \tau_{\mathrm{D}}$ in this process of average duration time $\tau_{\mathrm{D}}$ since the drift velocity is given by $F / \gamma=\beta F D_{0}$. Thus, the average displacement of the motor in a cycle is estimated as $\delta-\beta F D_{0} \tau_{\mathrm{D}}$, from which we have $F_{\mathrm{s}} \simeq \delta / \beta D_{0} \tau_{\mathrm{D}}$.

\section{Possibility of ATP synthesis}

Although KIF1A works as a transporter in cells, it would be interesting to ask if KIF1A can synthesize ATP when it is pulled backward by an external force. We have investigated this possibility and found that ATP can be synthesized in principle but it is practically impossible to detect the ATP synthesis by KIF1A. As explained in Appendix D, the rate of ATP synthesis is only on the order of $10^{-6} \mathrm{~s}^{-1}$ when KIF1A is pulled with velocity of about $1 \mu \mathrm{m} / \mathrm{s}$ under a typical condition for ATP synthesis: KIF1A needs to be pulled by a distance of $1 \mathrm{~m}$ to produce a single ATP molecule.

\section{ENERGETICS}

How good is the performance of KIF1A as a motor from the energetic point of view? In this section, we consider the thermodynamic efficiency, the power output, and a generalized efficiency to estimate the performance of KIF1A quantitatively. We shall investigate the dependence of these quantities on the nucleotide concentrations [ATP], [ADP], and $\left[\mathrm{P}_{\mathrm{i}}\right]$.

\section{A. Thermodynamic efficiency}

The efficiency of energy transduction can be measured by the thermodynamic efficiency $\eta$ defined by

$$
\eta=\frac{v F}{r \Delta \mu}
$$

where the numerator is the rate of the work done by the motor against the load $F$, and the denominator is the rate of the freeenergy consumption by the ATP hydrolysis. In Eq. (58), $v$ and $r$ can be calculated from the exact analytic expressions (29), and $\Delta \mu$ from Eq. (34).

In Fig. 7(a), we show the dependence of $\eta$ on the external load $F$ under a specific condition: $[\mathrm{ATP}]=21 \mu \mathrm{M},[\mathrm{ADP}]=$ $0.1 \mu \mathrm{M}$, and $\left[\mathrm{P}_{\mathrm{i}}\right]=1.6 \mathrm{mM}$. The maximum efficiency at a fixed set of nucleotide concentrations will be denoted by $\eta^{*}\left([\mathrm{ATP}],[\mathrm{ADP}],\left[\mathrm{P}_{\mathrm{i}}\right]\right)$; see Fig. 7(a). In Fig. 7(b), we show the dependence of $\eta$ on $F$ for other sets of nucleotide concentrations together with the one shown in Fig. 7(a). From the definition (58) of the efficiency $\eta$, it is obvious that $\eta$ is zero when $F=0$ and that it is also zero when the load is so large that the motor stalls (at $F=F_{\mathrm{s}}$ ). So somewhere between $F=0$ and $F=F_{\mathrm{s}}$, there must be an optimum, as we seen in Fig. 7. Furthermore, these convex-upward curves are well approximated by parabolas (i.e., quadratic functions of $F$ ), and, hence, the maximum efficiency $\eta^{*}$ is obtained at the half of the stall force, $F \simeq F_{\mathrm{s}} / 2$. This is because the chemical reaction rate $r$ is almost independent of $F$ as shown in Fig. 4 and the load dependence of velocity $v$ is approximated by a linear function of $F$ as in Eq. (55). Therefore, $\eta^{*}$ can be 

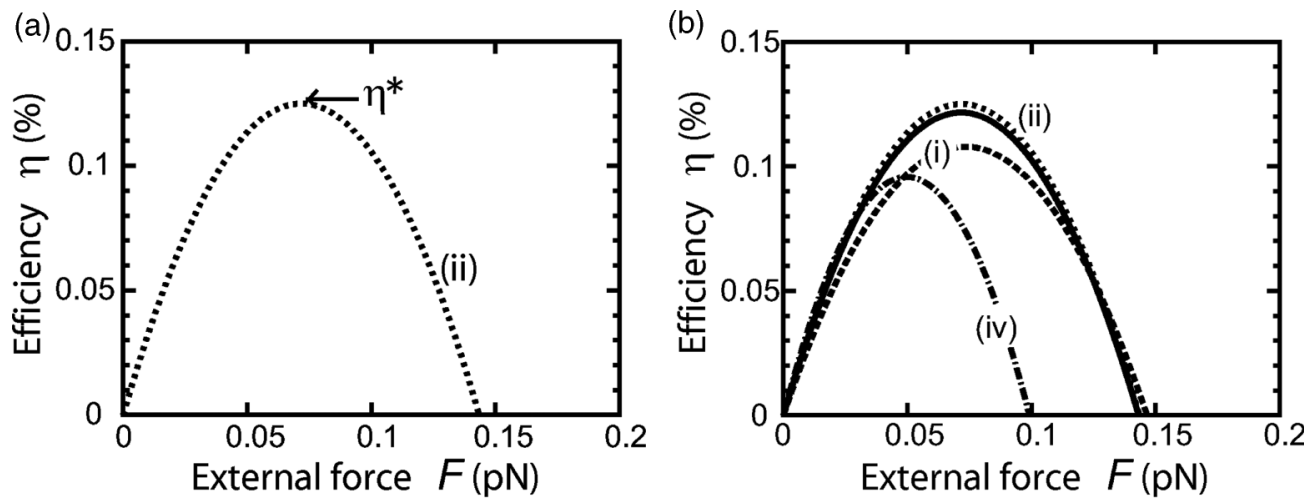

FIG. 7. The dependence of thermodynamic efficiency $\eta$ on external load $F$ for $\left[\mathrm{P}_{\mathrm{i}}\right]=1.6 \mathrm{mM}$. (a) The case with $([\mathrm{ATP}]$, [ADP]) $=$ $(21 \mu \mathrm{M}, 0.1 \mu \mathrm{M})$. The maximum value of $\eta$ for a given set of nucleotide concentrations is denoted by $\eta^{*}$. (b) The cases under other conditions, including the physiological one $([\mathrm{ATP}],[\mathrm{ADP}])=(2.0 \mathrm{mM}, 5.0 \mu \mathrm{M})$, represented by the solid line. The conditions for the curves with roman numbers i, ii, and iv are same as those used in Fig. 6: (i) ([ATP], [ADP] $)=(2.0 \mathrm{mM}, 0.1 \mu \mathrm{M})$ and (iv) $(2.0 \mathrm{mM}, 100 \mu \mathrm{M})$. The dotted curve (ii) is the same as the one in (a).

approximated by

$$
\eta^{*} \simeq \frac{F_{\mathrm{s}} \delta}{4 \Delta \mu},
$$

where the stall force $F_{\mathrm{s}}$ is given by the approximate expression Eq. (56), the characteristic displacement $\delta$ by Eq. (10), and the chemical potential difference $\Delta \mu$ by Eq. (34).

Now we consider the dependence of the maximum efficiency $\eta^{*}$ defined above on the nucleotide concentrations. Figure 8 demonstrates the dependence of $\eta^{*}$ on [ATP] and [ADP] for $\left[\mathrm{P}_{\mathrm{i}}\right]=1.6 \mathrm{mM}$ as a contour plot. In this example, the optimum value of $\eta^{*}$ is $\eta_{\max }^{*} \simeq 0.13 \%$, which is realized for ([ATP], $[\mathrm{ADP}]) \simeq(55 \mu \mathrm{M}, 1.3 \mu \mathrm{M})$. This optimal efficiency is about one-fourth of the one reported in Ref. [13], where the efficiency was roughly estimated by using expression $\eta=F_{\mathrm{s}} \delta / \Delta \mu$ instead of Eq. (59) with the values of $F_{\mathrm{s}}=$ $0.15 \mathrm{pN}$ and $\delta=3.6 \mathrm{~nm}$ measured in their experiment and $\Delta \mu \sim 25 k_{\mathrm{B}} T$ in the literature to obtain $\eta \sim 0.4 \%$. It is also noted that the optimal efficiency of about $0.1 \%$ for KIF1A is much smaller than the efficiency of $F_{1}$ motor $\left(F_{1}\right.$-ATPase); the latter was demonstrated to be almost $100 \%$ [25].

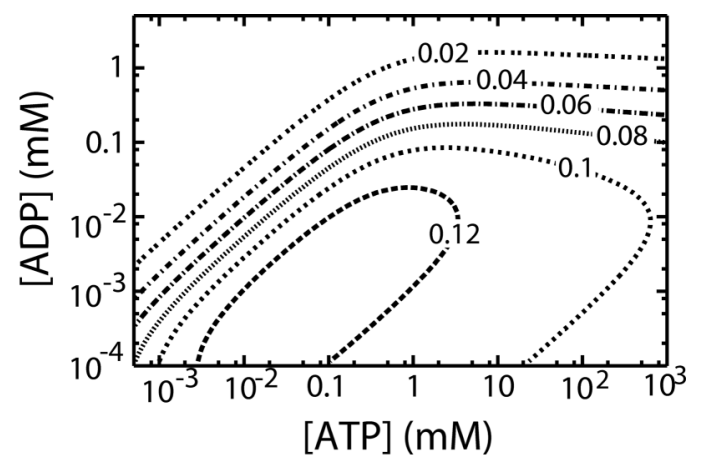

FIG. 8. Contour plot of the maximum efficiency $\eta^{*}$ on the ([ATP], $[\mathrm{ADP}]$ ) plane under the condition of $\left[\mathrm{P}_{\mathrm{i}}\right]=1.6 \mathrm{mM}$. The numbers attached to the contour lines indicate the values of $\eta^{*}$ in percentages.
The pattern of the contour lines of $\eta^{*}$ on the ([ATP], [ADP]) plane for other values of $\left[\mathrm{P}_{\mathrm{i}}\right]$ are similar to the one shown in Fig. 8. This is because $r$ and $v$ hardly change with the Pi concentration for $10^{-6} \mathrm{mM}<\left[\mathrm{P}_{\mathrm{i}}\right]<10^{2} \mathrm{mM}$, as explained in the preceding sections. Nevertheless, a weak dependence of the efficiency on the $\mathrm{P}_{\mathrm{i}}$ concentration arises through $\Delta \mu$ in Eq. (58), which depends on $\left[\mathrm{P}_{\mathrm{i}}\right]$ as expressed in Eq. (34). The optimal efficiency $\eta_{\max }^{*}$ together with the ATP and ADP concentrations that yield the optimal efficiency for a few choices of $\left[\mathrm{P}_{\mathrm{i}}\right]$ are listed in Table I. It may be worth remarking that the product of [ATP] and [ADP] under the optimal condition for the efficiency is independent of $\left[\mathrm{P}_{\mathrm{i}}\right]$ : [ATP] [ADP] $\simeq 69 \mu \mathrm{M}^{2}$.

The dependence of $\eta^{*}$ approximated by Eq. (59) on the nucleotide concentrations is explicitly given in Eq. (E5) of Appendix E. The value of the optimal efficiency $\eta_{\max }^{*}$ and the corresponding values of $[\mathrm{ATP}]$ and $[\mathrm{ADP}]$ for given $\left[\mathrm{P}_{\mathrm{i}}\right]$ estimated from this expression are presented in the parentheses of Table I. As one can see, these approximate results agree quite well with the exact ones.

\section{B. Power}

For biological activities, molecular machines may be required to work rapidly. The power, which is the mechanical work done by a machine per unit time, is a measure of how fast a machine does a useful work. The power $p$ of KIF1A moving with a constant velocity $v$ against an external load $F$ is given by

$$
p=F v .
$$

TABLE I. The optimal efficiency $\eta_{\max }^{*}$ and the ATP and ADP concentrations that yield $\eta_{\max }^{*}$ for a given concentration of $\mathrm{P}_{\mathrm{i}}$. The values in the parentheses are the results of approximate calculation explained in Appendix E.

\begin{tabular}{llcl}
\hline \hline$\left[\mathrm{P}_{\mathrm{i}}\right](\mathrm{mM})$ & \multicolumn{1}{c}{$\eta_{\max }^{*}(\%)$} & {$[\mathrm{ATP}](\mu \mathrm{M})$} & {$[\mathrm{ADP}](\mu \mathrm{M})$} \\
\hline $10^{-6}$ & $0.081(0.081)$ & $72(69)$ & $0.95(0.96)$ \\
1.6 & $0.13(0.13)$ & $55(53)$ & $1.3(1.3)$ \\
$10^{2}$ & $0.16(0.16)$ & $49(47)$ & $1.4(1.4)$ \\
\hline \hline
\end{tabular}



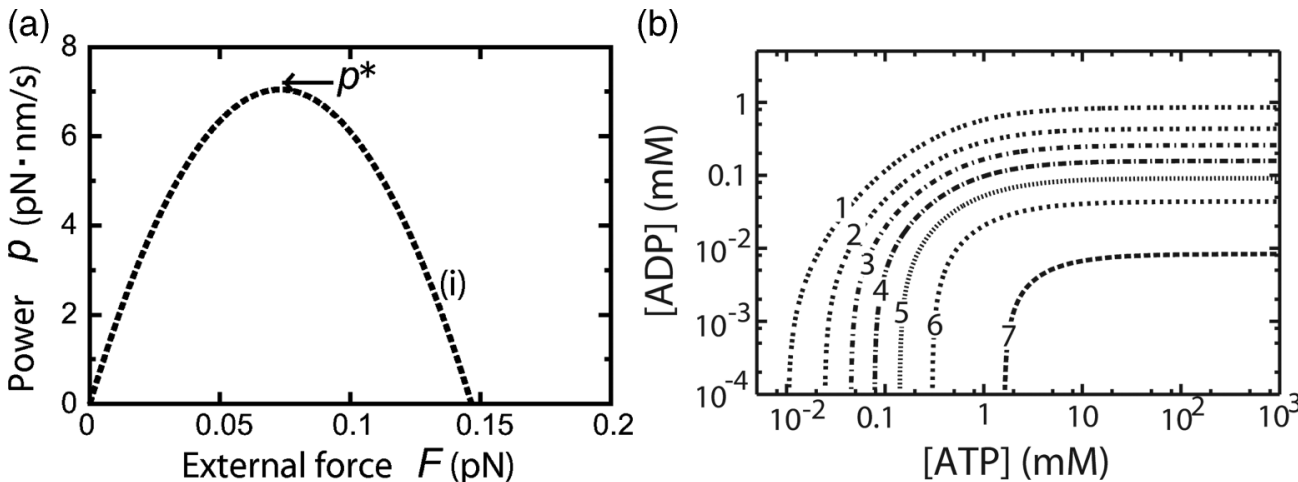

FIG. 9. (a) Dependence of power $p$ on the external force $F$ and the definition of the maximum power $p^{*}$ for $[\mathrm{ATP}]=2.0 \mathrm{mM}$, $[\mathrm{ADP}]=$ $0.1 \mu \mathrm{M}$, and $\left[\mathrm{P}_{\mathrm{i}}\right]=1.6 \mathrm{mM}$. The roman number $\mathrm{i}$ corresponds to the one used in Fig. 6 . (b) The contour plot of the maximum power $p^{*}$ on the ([ATP], $[\mathrm{ADP}])$ plane under the condition of $\left[\mathrm{P}_{\mathrm{i}}\right]=1.6 \mathrm{mM}$. The numbers attached to the contour lines indicate the values of $p^{*}$ in units of $\mathrm{pNnm} / \mathrm{s}$.

In this equation, $v$ can be calculated from the exact analytic expressions Eq. (29). In Fig. 9(a), we show the dependence of the power on the load, $p(F)$, for $[\mathrm{ATP}]=2.0 \mathrm{mM}$, [ADP $]=$ $0.1 \mu \mathrm{M}$, and $\left[\mathrm{P}_{\mathrm{i}}\right]=1.6 \mathrm{mM}$. The parabolic shape of the curve in this figure can be understood intuitively as in the case of efficiency $\eta$ shown in Fig. 7, and it is also concluded that this curve is well approximated by a parabola (quadratic function of $F$ ).

The maximum power at a fixed set of nucleotide concentrations is denoted by $p^{*}\left([\mathrm{ATP}],[\mathrm{ADP}],\left[\mathrm{P}_{\mathrm{i}}\right]\right)$; see Fig. 9(a). The dependence of the maximum power $p^{*}$ on [ATP] and [ADP] for $\left[\mathrm{P}_{\mathrm{i}}\right]=1.6 \mathrm{mM}$ is shown in Fig. 9(b) as a contour plot. The contour lines are almost parallel to the [ATP] axis in the limit of large [ATP] and to the [ADP] axis in the limit of small [ADP]. This pattern of the contour lines for $p^{*}$ significantly differs from the one for $\eta^{*}$ in Fig. 8. The upper limit of $p^{*}, p_{\max }^{*} \simeq 7.3 \mathrm{pN} \mathrm{nm} / \mathrm{s}$, is approached in the limit of high [ATP] and low [ADP]. The upper limit $p_{\max }^{*}$ hardly changes with $\left[\mathrm{P}_{\mathrm{i}}\right]$, because $v(F)$ is almost independent of $\left[\mathrm{P}_{\mathrm{i}}\right]$ for $10^{-6} \mathrm{mM}<\left[\mathrm{P}_{\mathrm{i}}\right]<10^{2} \mathrm{mM}$, as explained before.

The dependence of $p^{*}$ on the nucleotide concentrations discussed above can be described semiquantitatively by using the approximate expression $p^{*} \simeq F_{s} r \delta / 4$ obtained from Eqs. (55) and (60). The quantities $F_{\mathrm{s}}$ and $r$ in this expression are approximately given by Eqs. (56) and (36), respectively. From these approximate expressions we obtain Eq. (E4) in Appendix $\mathrm{E}$ for the upper limit of the power, which yields $p_{\max }^{*} \simeq 7.4 \mathrm{pN} \mathrm{nm} / \mathrm{s}$. This result agrees well with the exact one, $7.3 \mathrm{pN} \mathrm{nm} / \mathrm{s}$.

\section{Generalized efficiency}

Generally, motor proteins such as KIF1A that transport chemicals inside the cells operate in the absence of an external load. In order to evaluate the performance of molecular machines in such a situation, Derényi and Astumian [15] defined a generalized efficiency as the ratio of the minimum energy input required to accomplish the same task as the machine does to the actual input energy of the machine. In the case of KIF1A the task is to move with a velocity $v$, and the generalized efficiency is expressed as [15]

$$
\eta^{\mathrm{g}}=\frac{v^{2} /\left(D_{0} \beta\right)}{r \Delta \mu} \quad(F=0)
$$

where $1 /\left(D_{0} \beta\right)$ is equal to the friction coefficient of KIF1A and $v^{2} /\left(D_{0} \beta\right)$ is the energy dissipated per unit time by the translation of the motor. The generalized efficiency coincides with what is called the Stokes efficiency by Wang and Oster [16] for $F=0$.

Figure 10 demonstrates the dependence of $\eta^{\mathrm{g}}$ on [ATP] and $[\mathrm{ADP}]$ for $\left[\mathrm{P}_{\mathrm{i}}\right]=1.6 \mathrm{mM}$ as a contour plot, obtained from Eq. (61) by substituting the values of $v, r$, and $\Delta \mu$ numerically calculated using the exact expressions given in Eqs. (29) and (34). In this example, the optimum value of $\eta^{\mathrm{g}}$ is $\eta_{\max }^{\mathrm{g}}=0.11 \%$, which is realized for ([ATP], [ADP]) $=$ $(1.7 \mathrm{mM}, 45 \mu \mathrm{M})$. The pattern of the contour lines of $\eta^{\mathrm{g}}$ on the ([ATP], $[\mathrm{ADP}]$ ) plane for other values of $\left[\mathrm{P}_{\mathrm{i}}\right]$ is almost the same as the one shown in Fig. 10, for the same reason that explains the weak dependence of the similar contour plot of the maximum thermodynamic efficiency $\eta^{*}$ on $\left[\mathrm{P}_{\mathrm{i}}\right]$. A weak dependence of $\eta^{\mathrm{g}}$ on $\left[\mathrm{P}_{\mathrm{i}}\right]$ arises through $\Delta \mu$ in Eq. (61), which depends on $\left[\mathrm{P}_{\mathrm{i}}\right]$ as expressed in Eq. (34). The optimal efficiency $\eta_{\max }^{\mathrm{g}}$ together with the ATP and ADP concentrations that yield the optimal efficiency for a few choices of $\left[\mathrm{P}_{\mathrm{i}}\right]$ are listed in

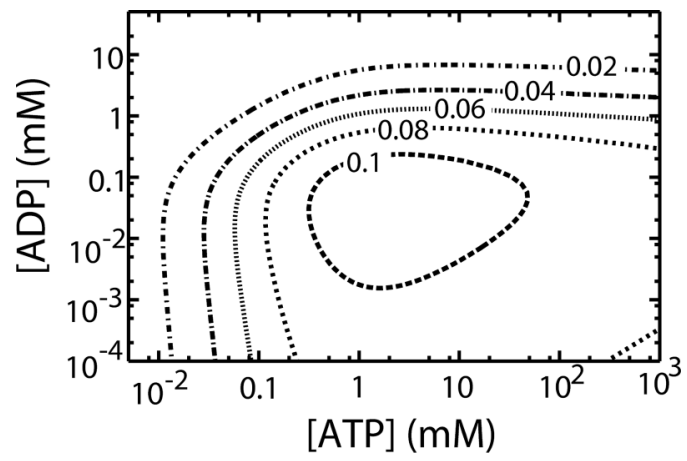

FIG. 10. The contour plot for generalized efficiency $\eta^{\mathrm{g}}$ on the ([ATP], $[\mathrm{ADP}]$ ) plane under the condition of $\left[\mathrm{P}_{\mathrm{i}}\right]=1.6 \mathrm{mM}$. The numbers attached to the contour lines indicate the values of $\eta^{\mathrm{g}}$ in percentages. 
TABLE II. The optimal efficiency $\eta_{\max }^{\mathrm{g}}$ and the ATP and ADP concentrations that yield $\eta_{\max }^{\mathrm{g}}$ for a given concentration of $\mathrm{P}_{\mathrm{i}}$. The values in the parentheses are the results of approximate calculation explained in Appendix E.

\begin{tabular}{llcc}
\hline \hline$\left[\mathrm{P}_{\mathrm{i}}\right](\mathrm{mM})$ & \multicolumn{1}{c}{$\eta_{\max }^{\mathrm{g}}(\%)$} & {$[\mathrm{ATP}](\mathrm{mM})$} & {$[\mathrm{ADP}](\mu \mathrm{M})$} \\
\hline $10^{-6}$ & $0.068(0.069)$ & $2.7(2.7)$ & $28(28)$ \\
1.6 & $0.11(0.11)$ & $1.7(1.7)$ & $45(45)$ \\
$10^{2}$ & $0.13(0.13)$ & $1.4(1.4)$ & $54(54)$ \\
\hline \hline
\end{tabular}

Table II. It may be worth remarking that the product of [ATP] and $[\mathrm{ADP}]$ under the optimal condition for the generalized efficiency $\eta^{\mathrm{g}}$ is independent of $\left[\mathrm{P}_{\mathrm{i}}\right]$ : [ATP] [ADP $]=7.5 \times$ $10^{-2} \mathrm{mM}^{2}$.

The dependence of $\eta^{\mathrm{g}}$ on the nucleotide concentrations discussed above can be described semiquantitatively by using the approximate expression $\eta^{\mathrm{g}} \simeq r \delta^{2} / D_{0} \beta \Delta \mu$ derived by substituting Eq. (52) into Eq. (61), where the reaction rate is approximated by Eq. (36). The value of the optimal efficiency $\eta_{\text {max }}^{\mathrm{g}}$ and the corresponding values of [ATP] and [ADP] can be estimated from this approximate expression for $\eta^{\mathrm{g}}$ as explained in Appendix E; these values are presented in the parentheses of Table II. As one can see, these approximate results agree quite well with the exact ones.

\section{KIF1A performs optimally under physiological conditions}

We have investigated the thermodynamic efficiency $\eta$, the power output $p$, and the generalized efficiency $\eta^{\mathrm{g}}$ separately. The maxima of $\eta$ and $p$ as functions of load $F$ for given nucleotide concentrations $[\mathrm{ATP}],[\mathrm{ADP}]$, and $\left[\mathrm{P}_{\mathrm{i}}\right]$ are denoted by $\eta^{*}$ and $p^{*}$, respectively. The dependencies of $\eta^{*}, p^{*}$, and $\eta^{\mathrm{g}}$ on [ATP] and [ADP] for $\left[\mathrm{P}_{\mathrm{i}}\right]=1.6 \mathrm{mM}$ are presented in Figs. 8, 9(b), and 10, respectively. From these data we have extracted the regions on the ([ATP], [ADP]) plane where conditions

$$
\eta^{*} \geqslant 0.9 \eta_{\max }^{*}, \quad p^{*} \geqslant 0.9 p_{\max }^{*}, \quad \eta^{\mathrm{g}} \geqslant 0.9 \eta_{\max }^{\mathrm{g}}
$$

are satisfied, respectively. The boundaries of these regions are presented in Fig. 11. One sees that there exists an area, the shaded region in this figure, on the ([ATP], [ADP]) plane where all the three conditions are satisfied. This area does not

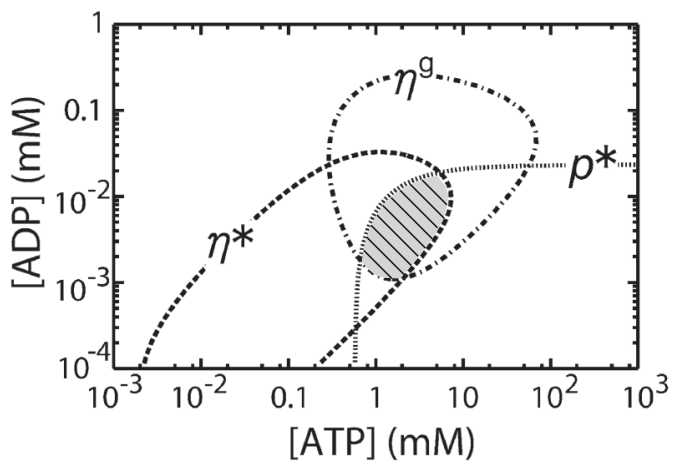

FIG. 11. The boundaries of three regions defined by inequalities in Eq. (62) on the ([ATP], $[\mathrm{ADP}])$ plane for $\left[\mathrm{P}_{\mathrm{i}}\right]=1.6 \mathrm{mM}$. The nucleotide concentrations in the shaded area satisfy these inequalities simultaneously. change very much upon the change in $\left[\mathrm{P}_{\mathrm{i}}\right]\left(10^{-6} \mathrm{mM} \leqslant\left[\mathrm{P}_{\mathrm{i}}\right] \leqslant\right.$ $\left.10^{2} \mathrm{mM}\right)$. This result demonstrates that KIF1A shows almost optimal performance all in $\eta, p$, and $\eta^{\mathrm{g}}$ if the concentrations of ATP, ADP, and $\mathrm{P}_{\mathrm{i}}$ are in this region. Furthermore, it is important to note that the nucleotide concentrations in cells ([ATP] $\sim 1 \mathrm{mM}$, [ADP] $\sim 10 \mu \mathrm{M}$, and $\left[\mathrm{P}_{\mathrm{i}}\right] \sim 1 \mathrm{mM}[2]$ ) lie in or near this region. It is likely that KIF1A has evolved to work efficiently from the energetic point of view under the physiological conditions.

\section{CONCLUSION}

In this article, we have introduced an elaborated model of the ratchet type for KIF1A, a single-headed biological molecular motor, to investigate how the chemical free energy is converted into mechanical work by taking into account the binding and release of reactant (ATP) and product (ADP and $\mathrm{P}_{\mathrm{i}}$ ) molecules to and from the motor explicitly. Using the model parameters determined so certain experimental results can be reproduced, the velocity and the reaction rate of the motor have been calculated under a wide variety of conditions concerning the external load force and the nucleotide concentrations. In particular, the dependence of the stall force $F_{\mathrm{s}}$ on the nucleotide concentrations has been clarified: Although $F_{\mathrm{s}}$ was found to be independent of the ATP concentration, [ATP], in the experiment of Ref. [13] with low ADP concentration, [ADP], we predict that the dependence of $F_{\mathrm{S}}$ on [ATP] and $[\mathrm{ADP}]$ can be observed if [ADP] is moderately large. We have also studied whether KIF1A can synthesize ATP if it is pulled backward and found that the synthesis is practically impossible though it is possible in principle. The energetic aspect of KIF1A has been investigated by evaluating the thermodynamic efficiency, the power output, and the generalized efficiency. It has turned out that the concentrations of ATP, ADP, and $\mathrm{P}_{\mathrm{i}}$ in cells yield more or less optimal values for all of these indices of energetic performance.

The optimal value of the thermodynamic efficiency, for example, is only about $0.1 \%$. In the present work the optimization with respect to nucleotide concentrations has been studied under the constraints that the other model parameters determined by considering the available experimental data be kept unchanged. The reason the optimal efficiency is so small should be the presence of the diffusion process (state D), which is necessary for the "single-headed" motor to move along the track by getting out of a potential well. Since the motor is pulled backward without much difficulty by an external load in state $\mathrm{D}$, it stalls under a small load force and cannot produce much work against the load. Hence, we have quite small efficiency. To overcome this problem dimeric kinesin (such as kinesin-1) should have been invented by the evolution so a head bound to the track prevents a detached or weakly bound head from being pulled backward without limitation under the load. The poor efficiency in a one-headed motor and its improvement by a two-headed mechanism were noticed in earlier works $[17,26]$ based on Brownian-ratchet models that mimic one-headed and two-headed molecular motors.

In the present work, we have studied the energetics of KIF1A moving alone. However, in vivo (in the cell) KIF1A normally conveys cargos (vesicles), and in some experiments [13] a latex bead is linked to KIF1A. Since a cargo or a 
bead (100 nm-1 $\mu \mathrm{m}$ in diameter) is significantly larger than $\operatorname{KIF} 1 \mathrm{~A}(\sim 4 \mathrm{~nm})$, the former should affect the motility and the energetics of the latter. Actually, a molecular dynamics study of KIF1A using a structure-based coarse-grained model [27] demonstrated that the large frictional cargo which is attached to KIF1A through a flexible linker can enhance the biased movement of the Brownian motion. The effects of a cargo linked to a simple Brownian ratchet on the motility and the generalized efficiency were also studied [28-30]. It would be interesting to extend the present model to include a cargo linked to KIF1A and investigate how the frictional coefficient of the cargo and the stiffness of the linker affect the energetic properties of KIF1A.

\section{ACKNOWLEDGMENTS}

We thank Y. Okada, H. Higuchi, and S. Takada for informative discussions at various stages of the present work. This work was supported in part by the Grants-in-Aid for Scientific Research in Priority Areas from the Ministry of Education, Culture, Sports, Science and Technology of Japan.

\section{APPENDIX A: DIFFUSION AND BIASED BINDING}

This appendix provides supplementary information on the qualitative discussions of Secs. II B and III A. We will give detailed explanations of (i) the diffusion process in state D, (ii) the relation of parameter $\delta_{1}$ to the displacement associated with the biased binding observed in Ref. [13], and (iii) the average transition rate from state $D$ to state $\Phi$. It will be also shown that the stall force discussed in Sec. IV B can be estimated in a certain extreme condition by extending the qualitative argument to include the effect of load force. The argument given here is a slightly modified and somewhat detailed version of the theoretical treatment in Supplementary Information of Ref. [13].

\section{Diffusion process}

We shall show that the average displacement of motor from the moment of transition $\mathrm{T} \rightarrow \mathrm{D}$ to the moment of transition $\mathrm{D} \rightarrow \Phi$ is zero. If the transition to state $\Phi$ occurs everywhere along the MT, the average displacement will obviously be zero. However, the transition to state $\Phi$ is possible only at restricted positions in the present model. In this case, it is not so trivial whether the average displacement is zero.

Suppose that a transition from state $\mathrm{T}$ to state $\mathrm{D}$ takes place at time $t=0$ and at position $x=x_{\mathrm{P}}$ (see Fig. 3). The motor undergoes diffusive motion in state $\mathrm{D}$, and the probability distribution of motor position $x$ at time $t>0$ will be

$$
P(x, t)=\frac{1}{\sqrt{4 \pi D_{0} t}} \exp \left[-\frac{\left(x-x_{\mathrm{P}}\right)^{2}}{4 D_{0} t}\right],
$$

where $D_{0}$ is the diffusion coefficient. Since the transition $\mathrm{D} \rightarrow$ $\Phi$ is possible only at positions $x_{n}=x_{\mathrm{P}}-\delta+n L$ with $n$ being integers, the value of the displacement during state $\mathrm{D}$ takes on

$$
\Delta x_{n}=x_{n}-x_{\mathrm{P}}=n L-\delta \text {. }
$$

Hence, the average displacement is given by

$$
\Delta x=\sum_{n=-\infty}^{\infty} \Delta x_{n} P\left(x_{n}, t\right) / \sum_{n=-\infty}^{\infty} P\left(x_{n}, t\right)
$$

if the transition to state $\Phi$ occurs at time $t$.

The sums in Eq. (A3) can be estimated by making use of Poisson's summation formula. It is not difficult to see that they can be approximated by

$$
L \sum_{n=-\infty}^{\infty} P\left(x_{n}\right) \simeq 1, \quad \sum_{n=-\infty}^{\infty} \Delta x_{n} P\left(x_{n}\right) \simeq 0
$$

with high precision provided that $2 D_{0} t / L^{2}>1$ : The errors are about $10^{-8}$ or less. If we substitute the average lifetime of state D into $t$, we have $2 D_{0} t / L^{2} \simeq 3$ for $\mathrm{KIF} 1 \mathrm{~A}\left(t \simeq 4 \mathrm{~ms}, D_{0} \simeq\right.$ $2.6 \times 10^{4} \mathrm{~nm}^{2} / \mathrm{s}$, and $L=8 \mathrm{~nm}$; see Sec. III B). Therefore, the approximation of Eq. (A4) is valid and it is concluded that $\Delta x$ given in Eq. (A3) is zero.

\section{Biased binding}

Okada and coworkers [13] reported that KIF1A make a translational movement upon transition from state $\mathrm{D}$ to state $\Phi$. In their experiment KIF1A was attached to a bead, and the bead was trapped by an optical trap. The average locations of the bead before and after the transition $\mathrm{D} \rightarrow \Phi$ (i.e., the binding of KIF1A to the MT) were measured to determine the displacement associated with this transition. The average displacement was found to be $\delta_{\text {bind }}=2.8 \pm 0.8 \mathrm{~nm}$. Thus, the biased movement of KIF1A toward the plus end of the MT upon binding to the MT was revealed.

This biased binding is explained by the present model as follows. The probability distribution of motor's position $x$ in the weak-binding state (state D) is given by

$$
P_{\mathrm{eq}}(x)=\sqrt{\frac{K}{2 \pi k_{\mathrm{B}} T}} \exp \left[-\frac{K\left(x-x_{0}\right)^{2}}{2 k_{\mathrm{B}} T}\right],
$$

where $K$ is the stiffness of the optical trap and $x_{0}$ is the position of the motor when the bead sits at the trap center. The binding of the motor takes place at one of locations $x_{n}=x_{D}+n L$ with $n$ being integers. Right after the binding, the motor slides down the slope of potential $V_{\Phi}$ by distance $\delta_{1}$ (see Fig. 3). Since the average position of the motor before the transition is $x_{0}$, the displacement of the motor upon binding is given by

$$
\Delta x_{n}=x_{n}+\delta_{1}-x_{0}
$$

when the transition occurs at $x_{n}$. Therefore, the average displacement $\Delta x$ is calculated as

$$
\Delta x=\sum_{n=-\infty}^{\infty} \Delta x_{n} P_{\mathrm{eq}}\left(x_{n}\right) / \sum_{n=-\infty}^{\infty} P_{\mathrm{eq}}\left(x_{n}\right)
$$

The sums in this expression again can be estimated by using Poisson's summation formula to obtain

$$
L \sum_{n=-\infty}^{\infty} P_{\mathrm{eq}}\left(x_{n}\right) \simeq 1, \quad \sum_{n=-\infty}^{\infty} \Delta x_{n} P_{\mathrm{eq}}\left(x_{n}\right) \simeq \frac{\delta_{1}}{L},
$$

for $k_{\mathrm{B}} T / K L^{2}>1$ with errors of about $10^{-8}$ or less. For the experimental setup of Ref. [13] we have $k_{\mathrm{B}} T / K L^{2} \sim 10$ and 
the above approximation is verified, which indicates

$$
\Delta x \simeq \delta_{1} .
$$

Therefore, the parameter $\delta_{1}$ in our model corresponds to the displacement $\delta_{\text {bind }}$ associated with the biased binding observed by Okada et al. [13].

\section{ADP release rate}

If the probability distribution of the motor position $x$ at time $t$ in state $\mathrm{D}$ is given by Eq. (A1), the transition rate from state $\mathrm{D}$ to state $\Phi$ (the rate of ADP release) at time $t$ is given by

$$
\Omega_{\Phi \mathrm{D}}=\int_{-\infty}^{\infty} \omega_{\Phi \mathrm{D}}(x) P(x, t) d x .
$$

Substitution of Eqs. (13) and (A1) into this equation yields

$$
\Omega_{\Phi \mathrm{D}}=k_{\mathrm{D}}^{u} \sum_{n=-\infty}^{\infty} P\left(x_{n}, t\right) \simeq k_{\mathrm{D}}^{u} / L,
$$

where we have assumed that $2 D_{0} t / L^{2}>1$ and used the first relation in Eq. (A4). Note that rate $\Omega_{\Phi \mathrm{D}}$ is independent of time $t$. Therefore, the average waiting time for the ADP release is given by

$$
\tau=1 / \Omega_{\Phi \mathrm{D}} \simeq L / k_{\mathrm{D}}^{u}
$$

\section{Stall force}

Extending the analysis of Appendix A 1 to the situation in which a constant load $F$ is applied to the motor, we can obtain a rough estimate of the stall force. In the presence of the load, the motor in state $\mathrm{D}$ drifts to the negative direction of the $x$ axis by distance $F t / \gamma$ on average in time $t$, where $\gamma=k_{\mathrm{B}} T / D_{0}$ is the frictional drag coefficient of the motor. Hence, the probability distribution of motor position $x$ at time $t$ is now given by

$$
P(x, t)=\frac{1}{\sqrt{4 \pi D_{0} t}} \exp \left[-\frac{\left(x-x_{\mathrm{P}}+F t / \gamma\right)^{2}}{4 D_{0} t}\right]
$$

instead of Eq. (A1). If the transition from state D to state $\Phi$ occurs at time $t$, the average displacement $\Delta x(t)$ at this moment is obtained by substituting Eq. (A13) into Eq. (A3). Carrying out the summations in Eq. (A3) as before, we have

$$
\Delta x(t) \simeq-F t / \gamma
$$

with sufficient accuracy. Averaging Eq. (A14) over transition time $t$ yields the average displacement $\Delta x \simeq-F \tau / \gamma$ of the motor from the moment of transition $\mathrm{T} \rightarrow \mathrm{D}$ to the moment of transition $D \rightarrow \Phi$, where $\tau \simeq L / k_{\mathrm{D}}^{\mathrm{u}}$ is the average lifetime of state D.

As explained in Sec. II B, the motor translates by distance $\delta_{1}$ right after a transition $\mathrm{D} \rightarrow \Phi$ and by distance $\delta_{2}$ right after a transition $\Phi \rightarrow \mathrm{T}$. Therefore, the average displacement of motor per one ATP hydrolysis cycle is estimated as

$$
\Delta x_{\text {cycle }}=\delta_{1}+\delta_{2}+\Delta x \simeq \delta-F \tau / \gamma,
$$

where $\delta=\delta_{1}+\delta_{2}$. Let $r$ be the rate of ATP hydrolysis. We then obtain

$$
v=r \Delta x_{\text {cycle }} \simeq\left(1-F / F_{\mathrm{s}}\right) r \delta
$$

for the average velocity $v$, where the stall force is given by $F_{\mathrm{s}} \simeq \gamma \delta / \tau=k_{\mathrm{B}} T \delta / D_{0} \tau$.

\section{APPENDIX B: EXACT SOLUTION OF FOKKER-PLANCK EQUATION}

\section{Integration of the Fokker-Planck equation}

This appendix explains how the Fokker-Planck equation is solved analytically in the case of steady state. In order to simplify the expressions given below, integer indices $i=0,1,2$ will be assigned for the $\Phi, \mathrm{T}$, and D states, respectively. It is also convenient to introduce two-index notations $x_{i}^{n}$ and $\omega_{i}^{n}$ for the locations of transitions and transition rates, respectively, defined as

$x_{0}^{1}=x_{1}^{0}=x_{\mathrm{T}}, \quad x_{1}^{1}=x_{2}^{0}=x_{\mathrm{P}}, \quad x_{2}^{1}=x_{0}^{0}-L=x_{\mathrm{D}}$

and

$$
\begin{aligned}
& \omega_{0}^{1}=k_{\mathrm{T}}^{\mathrm{b}}, \quad \omega_{1}^{0}=k_{\mathrm{T}}^{\mathrm{u}}, \quad \omega_{1}^{1}=k_{\mathrm{P}}^{\mathrm{u}}, \quad \omega_{2}^{0}=k_{\mathrm{P}}^{\mathrm{b}}, \\
& \omega_{2}^{1}=k_{\mathrm{D}}^{\mathrm{u}}, \quad \omega_{0}^{0}=k_{\mathrm{D}}^{\mathrm{b}} .
\end{aligned}
$$

For other integer values of $i$ and $n$ than those shown in these equations, $x_{i}^{n}$ and $\omega_{i}^{n}$ are defined through the following periodicity relation

$$
x_{i}^{n}=x_{i+3}^{n}=x_{i}^{n+2}-L, \quad \omega_{i}^{n}=\omega_{i}^{n+2}=\omega_{i+3}^{n} .
$$

In the steady-state situation, the Fokker-Planck equations (21) read

$$
\begin{gathered}
\frac{d J_{j}}{d x}=\sum_{i=0}^{2}\left[-\omega_{i j} P_{j}+\omega_{j i} P_{i}\right], \\
J_{j}=-D_{0}\left(\frac{d}{d x}+\frac{d U_{j}}{d x}\right) P_{j},
\end{gathered}
$$

for $j=0,1,2$. Because the transition rates $\omega_{i j}(x)$ are sums of the $\delta$ functions, Eq. (B4) implies that the probability current $J_{i}(x)$ is constant in the interval

$$
I_{i}^{n}=\left(x_{i}^{n}, x_{i}^{n+1}\right) \text {. }
$$

This constant value will be denoted by $J_{i}^{n}$, i.e.,

$$
J_{i}^{n}=J_{i}(x) \text { for } x \in I_{i}^{n} .
$$

Note that we have $J_{i}^{n \pm 2}=J_{i}^{n}$ from the periodicity of $J_{i}(x)$.

Now, integrating Eq. (B5) in the interval $I_{i}^{n}$, we obtain

$$
\begin{aligned}
P_{i}(x)= & D_{0} P_{i}^{n} E_{i}\left(x_{i}^{n}, x\right) \\
& -J_{i}^{n} \int_{x_{i}^{n}}^{x} E_{i}\left(x^{\prime}, x\right) d x^{\prime} \quad \text { for } \quad x \in I_{i}^{n},
\end{aligned}
$$

where constants $P_{i}^{n}$ are defined by

$$
P_{i}^{n}=P_{i}\left(x_{i}^{n}\right)
$$

and function $E_{i}\left(x, x^{\prime}\right)$ by

$$
E_{i}\left(x, x^{\prime}\right)=\left(1 / D_{0}\right) \exp \left[U_{i}(x)-U_{i}\left(x^{\prime}\right)\right] .
$$

The periodicity of $P_{i}(x)$ implies that $P_{i}^{n \pm 2}=P_{i}^{n}$.

By substituting $x=x_{i}^{n+1}$ into Eq. (B8), the following expression for $J_{i}^{n}$ in terms $P_{i}^{n}$ can be obtained:

$$
J_{i}^{n}=\alpha_{i}^{n} P_{i}^{n}-\beta_{i}^{n+1} P_{i}^{n+1},
$$


where

and

$$
\alpha_{i}^{n}=\left[\int_{x_{i}^{n}}^{x_{i}^{n+1}} E_{i}\left(x, x_{i}^{n}\right) d x\right]^{-1}
$$

$$
\beta_{i}^{n}=\alpha_{i}^{n-1} \exp \left[U_{i}\left(x_{i}^{n}\right)-U_{i}\left(x_{i}^{n-1}\right)\right]
$$

are positive constants determined by the effective potentials $U_{i}(x)$ and the diffusion coefficient $D_{0}$. Equations (B8) and (B11) indicate that the knowledge of the integration constants $P_{i}^{n}$ enables us obtain the solution of the FokkerPlanck equation. It is enough to know six $P_{i}^{n}$ with the pairs of indices $i$ and $n$ that appear in Eq. (B1) due to the periodicity of $P_{i}(x)$. These constants are obtained as explained in the next paragraph.

From Eq. (B11) and the equation obtained by integrating Eq. (B4) from $x=x_{i}^{n}-0$ to $x=x_{i}^{n}+0$, we obtain

$$
\left(\gamma_{i}^{n}+\omega_{i}^{n}\right) P_{i}^{n}-\gamma_{i}^{n \pm 1} P_{i}^{n \pm 1}-\omega_{i \mp 1}^{n \pm 1} P_{i \mp 1}^{n \pm 1}=0,
$$

where the $\gamma_{i}^{n}$ is defined by

$$
\gamma_{i}^{n}=\alpha_{i}^{n}+\beta_{i}^{n} .
$$

In Eq. (B14), the upper and lower signs are for $n$ even and odd, respectively. It is not difficult to see that, of six equations obtained from Eq. (B14) with $i$ and $n$ that appear in Eq. (B1), only five are independent. Another equation for determining $P_{i}^{n}$ comes from the normalization condition (25). Substitution of Eq. (B8) into Eq. (25) yields

$$
\sum_{i=0}^{2} \sum_{n=0}^{1} \psi_{i}^{n} P_{i}^{n}=1
$$

where the positive constant $\psi_{i}^{n}$ is defined by

$$
\psi_{i}^{n}=\xi_{i}^{n} \alpha_{i}^{n}+\eta_{i}^{n-1} \beta_{i}^{n}
$$

with

$$
\begin{gathered}
\xi_{i}^{n}=\int_{x_{i}^{n}}^{x_{i}^{n+1}} d x \int_{x}^{x_{i}^{n+1}} E_{i}\left(x^{\prime}, x\right) d x^{\prime}, \\
\eta_{i}^{n}=\int_{x_{i}^{n}}^{x_{i}^{n+1}} d x \int_{x_{i}^{n}}^{x} E_{i}\left(x^{\prime}, x\right) d x^{\prime} .
\end{gathered}
$$

It is straightforward to obtain $P_{i}^{n}$ by solving the linear algebraic equations (B14) together with (B16).

\section{Velocity and reaction rate}

Once $P_{i}^{n}$ are obtained as explained in the proceeding subsection, the average velocity $v$ and the average reaction rate $r$ can be calculated according to

$$
\begin{gathered}
v=\left(\alpha_{0}^{1} P_{0}^{1}+\alpha_{1}^{1} P_{1}^{1}+\alpha_{2}^{0} P_{2}^{0}-\beta_{0}^{0} P_{0}^{0}-\beta_{1}^{0} P_{1}^{0}-\beta_{2}^{1} P_{2}^{1}\right) L, \\
r=\omega_{0}^{1} P_{0}^{1}-\omega_{1}^{0} P_{1}^{0},
\end{gathered}
$$

where the former is obtained from Eqs. (27) and (B11) and the latter from Eq. (28). The results obtained by substituting $P_{i}^{n}$ into Eqs. (B20) and (B21) may be expressed as Eq. (29) in the text together with Eqs. (30)-(32). The coefficients $C_{l m n}, \sigma$, and $\lambda_{l m n}$ in the latter equations are expressed by messy equations. In order to reduce the messiness, we introduce single-index notations $\alpha_{v}, \beta_{v}$, etc., in place of $\alpha_{i}^{n}, \beta_{i}^{n}$, etc., where $v$ is related with $i$ and $n$ through $v=2 i+n$, i.e., we have

$$
\begin{aligned}
& \alpha_{0}=\alpha_{0}^{0}, \quad \alpha_{1}=\alpha_{0}^{1}, \quad \alpha_{2}=\alpha_{1}^{0}, \quad \alpha_{3}=\alpha_{1}^{1}, \\
& \alpha_{4}=\alpha_{2}^{0}, \quad \alpha_{5}=\alpha_{2}^{1},
\end{aligned}
$$

and similar expressions for $\beta_{v}, \gamma_{v}$, and $\psi_{v}$. Now $C_{l m n}$ are given by

$$
\begin{gathered}
C_{000}=\left(\psi_{0} \frac{\gamma_{1}}{\gamma_{0}}+\psi_{1}\right)\left(\frac{1}{\gamma_{2}}+\frac{1}{\kappa_{\mathrm{T}}}+\frac{\gamma_{3}}{\gamma_{2} \kappa_{\mathrm{P}}}\right), \\
C_{100}=\left(\psi_{2} \frac{\gamma_{3}}{\gamma_{2}}+\psi_{3}\right) \frac{1}{\kappa_{\mathrm{P}}}+\left(\psi_{4} \frac{\gamma_{5}}{\gamma_{4}}+\psi_{5}\right) \frac{1}{\kappa_{\mathrm{D}}} \\
+\frac{\psi_{0}}{\gamma_{0}}+\frac{\psi_{2}}{\gamma_{2}}+\frac{\psi_{4}}{\gamma_{4}}, \\
C_{010}=\frac{\gamma_{1}}{\gamma_{0}}\left(\psi_{4} \frac{\gamma_{5}}{\gamma_{4}}+\psi_{5}\right)\left(\frac{1}{\gamma_{2}}+\frac{1}{\kappa_{\mathrm{T}}}+\frac{\gamma_{3}}{\gamma_{2} \kappa_{\mathrm{P}}}\right), \\
C_{001}=\frac{\gamma_{3}}{\gamma_{2}}\left(\psi_{0} \frac{\gamma_{1}}{\gamma_{0}}+\psi_{1}\right)\left(\frac{1}{\gamma_{4}}+\frac{\gamma_{5}}{\gamma_{4} \kappa_{\mathrm{D}}}\right), \\
C_{110}=\frac{1}{\gamma_{0}}\left(\psi_{4} \frac{\gamma_{5}}{\gamma_{4}}+\psi_{5}\right), \\
C_{101}=\left(\psi_{2} \frac{\gamma_{3}}{\gamma_{2}}+\psi_{3}\right)\left(\frac{1}{\gamma_{4}}+\frac{\gamma_{5}}{\gamma_{4} \kappa_{\mathrm{D}}}\right), \\
C_{011}=\frac{\psi_{1} \gamma_{3} \gamma_{5}+\gamma_{1} \psi_{3} \gamma_{5}+\gamma_{1} \gamma_{3} \psi_{5}}{\gamma_{0} \gamma_{2} \gamma_{4}} \\
+\frac{\gamma_{1} \gamma_{5}}{\gamma_{0} \gamma_{4} \kappa_{T}}\left(\psi_{2} \frac{\gamma_{3}}{\gamma_{2}}+\psi_{3}\right), \\
C_{111}=\frac{\gamma_{5}}{\gamma_{0} \gamma_{4}}\left(\psi_{2} \frac{\gamma_{3}}{\gamma_{2}}+\psi_{3}\right) .
\end{gathered}
$$

The expression for $\sigma$ is much simpler:

$$
\sigma=\frac{\gamma_{1} \gamma_{3} \gamma_{5}}{\gamma_{0} \gamma_{2} \gamma_{4}} .
$$

Finally, $\lambda_{\operatorname{lmn}}$ are as follows:

$$
\begin{gathered}
\lambda_{000}=-\frac{\beta_{0} \beta_{1} G(F)}{\gamma_{0}}\left(\frac{1}{\gamma_{2}}+\frac{1}{\kappa_{\mathrm{T}}}+\frac{\gamma_{3}}{\gamma_{2} \kappa_{\mathrm{P}}}\right), \\
\lambda_{100}=\frac{\gamma_{0} \alpha_{2} \alpha_{4}-\gamma_{0} \beta_{2} \beta_{4}-\beta_{0} \gamma_{2} \gamma_{4}}{\gamma_{0} \gamma_{2} \gamma_{4}} \\
-G(F)\left(\frac{\beta_{2} \beta_{3}}{\gamma_{2} \kappa_{\mathrm{P}}}+\frac{\beta_{4} \beta_{5}}{\gamma_{4} \kappa_{\mathrm{D}}}\right), \\
\lambda_{010}=-\frac{\beta_{4} \beta_{5} \gamma_{1} G(F)}{\gamma_{4} \gamma_{0}}\left(\frac{1}{\gamma_{2}}+\frac{1}{\kappa_{\mathrm{T}}}+\frac{\gamma_{3}}{\gamma_{2} \kappa_{\mathrm{P}}}\right), \\
\lambda_{001}=-\frac{\beta_{0} \beta_{1} \gamma_{3} G(F)}{\gamma_{0} \gamma_{2}}\left(\frac{1}{\gamma_{4}}+\frac{\gamma_{5}}{\gamma_{4} \kappa_{\mathrm{D}}}\right), \\
\lambda_{110}=-\frac{\beta_{4} \beta_{5} G(F)}{\gamma_{4} \gamma_{0}}, \\
\lambda_{101}=-\frac{\beta_{2} \beta_{3} G(F)}{\gamma_{2}}\left(\frac{1}{\gamma_{4}}+\frac{\gamma_{5}}{\gamma_{4} \kappa_{\mathrm{D}}}\right), \\
\lambda_{011}=-\frac{\gamma_{1} \beta_{3} \beta_{5}-\gamma_{1} \alpha_{3} \alpha_{5}-\alpha_{1} \gamma_{3} \gamma_{5}}{\gamma_{0} \gamma_{2} \gamma_{4}}-\frac{\gamma_{1} \gamma_{5} \beta_{2} \beta_{3} G(F)}{\gamma_{0} \gamma_{4} \kappa_{\mathrm{T}}}, \\
\lambda_{111}=-\frac{\gamma_{5} \beta_{2} \beta_{3} G(F)}{\gamma_{0} \gamma_{2} \gamma_{4}},
\end{gathered}
$$


where function $G(F)$ is defined by

$$
G(F)=1-\exp \left(-F L / k_{\mathrm{B}} T\right),
$$

and, hence, $G=0$ for $F=0$. Note that $C_{l m n}$ have the dimensions of time, while $\sigma$ and $\lambda_{\operatorname{lm} n}$ are dimensionless. It is also noted that $C_{l m n}$ and $\sigma$ are positive, whereas $\lambda_{l m n}$ can be positive or negative.

\section{Definite integrals}

The integrals $\alpha_{i}^{n}, \xi_{i}^{n}$, and $\eta_{i}^{n}$ defined in Eqs. (B12), (B18), and (B19), respectively, can be carried out analytically for piecewise linear functions $U_{i}(x)$. These integrals are building blocks of $\alpha_{v}, \beta_{v}, \gamma_{v}, \psi_{v}$ in Eqs. (B23)-(B39); see Eqs. (B13), (B15), and (B17). In order to express these integrals concisely, we introduce new constants,

$$
l_{i}^{n}=x_{i}^{n+1}-x_{i}^{n}, \quad u_{i}^{n}=U_{i}\left(x_{i}^{n+1}\right)-U_{i}\left(x_{i}^{n}\right),
$$

and new functions,

$$
\begin{gathered}
g(x)=\frac{\exp (x)-1}{x}, \\
h(x)=\frac{1}{x}\left[\frac{\exp (x)-1}{x}-1\right] .
\end{gathered}
$$

Note that the definition of $\beta_{i}^{n}$ (B13) may be rewritten as $\beta_{i}^{n}=$ $\alpha_{i}^{n-1} \exp \left(u_{i}^{n-1}\right)$.

In the cases $(i, n)=(0,0),(1,0),(2,0)$, and $(2,1)$, definite integrals $\alpha_{i}^{n}, \xi_{i}^{n}$, and $\eta_{i}^{n}$ are given by

$$
\begin{gathered}
\alpha_{i}^{n}=D_{0} / g\left(u_{i}^{n}\right) l_{i}^{n}, \\
\xi_{i}^{n}=h\left(u_{i}^{n}\right)\left(l_{i}^{n}\right)^{2} / D_{0}, \\
\eta_{i}^{n}=h\left(-u_{i}^{n}\right)\left(l_{i}^{n}\right)^{2} / D_{0} .
\end{gathered}
$$

In the other cases $(i, n)=(0,1),(1,1)$, they are given by

$$
\begin{gathered}
\alpha_{i}^{1}=D_{0} /\left[g\left(v_{i}\right) y_{i}+\exp \left(u_{i}^{1}\right) g\left(w_{i}\right) z_{i}\right], \\
\xi_{i}^{1}=\frac{1}{D_{0}}\left[h\left(v_{i}\right)\left(y_{i}\right)^{2}+g\left(v_{i}\right) g\left(-w_{i}\right) y_{i} z_{i}+h\left(-w_{i}\right)\left(z_{i}\right)^{2}\right], \\
\eta_{i}^{1}=\frac{1}{D_{0}}\left[h\left(-v_{i}\right)\left(y_{i}\right)^{2}+g\left(-v_{i}\right) g\left(w_{i}\right) y_{i} z_{i}+h\left(w_{i}\right)\left(z_{i}\right)^{2}\right],
\end{gathered}
$$

where $y_{i}$ and $z_{i}$ are defined by

$$
y_{i}=a, \quad z_{i}=L-\delta_{i+1}-a,
$$

and $v_{i}$ and $w_{i}$ by

$$
v_{i}=\beta(W+F a), \quad w_{i}=\beta\left(\frac{W}{L-a}-F\right) z_{i} .
$$

We find that $\gamma_{4}$ and $\gamma_{5}$ are identical, i.e.,

$$
\gamma_{4}=\gamma_{5}=\frac{D_{0} g(\beta F)}{g\left(u_{2}^{0}\right) g\left(u_{2}^{1}\right) l_{2}^{0} l_{2}^{1}},
$$

by substituting Eqs. (B44) and (B13) with $(i, n)=(2,0)$ and $(2,1)$ into Eq. (B15). This relation is useful because it can be used to simplify many of Eqs. (B23)-(B39), where one finds the ratio $\gamma_{5} / \gamma_{4}$, which has turned out to be unity, in various places.

\section{APPENDIX C: CERTAIN LIMITING CASES}

Here, we derive simplified expressions for the reaction rate $r$ and the velocity $v$ in certain limiting cases.

\section{Reaction rate for $F=0$}

When the external load $F$ is zero, we have $u_{0}^{0}=-u_{0}^{1}$, $u_{1}^{0}=-u_{1}^{1}$, and $u_{2}^{0}=u_{2}^{1}=0$ from Eqs. (B41) and (23). The first two relations lead to

$$
\gamma_{1} / \gamma_{0}=e^{-u_{0}^{1}}, \quad \gamma_{3} / \gamma_{2}=e^{-u_{1}^{1}} .
$$

Hence, $\sigma$ defined in Eq. (B31) are given by

$$
\sigma=\exp \left(-\frac{\beta W \delta}{L-a}\right),
$$

from which we obtain Eq. (33) for $Z_{r}$ in the text.

In the rest of this subsection, we assume that condition

$$
\exp (-\beta \Delta \mu) \ll 1
$$

is satisfied, so the reaction rate is approximated by

$$
r \simeq \rho_{\mathrm{T}} / R
$$

In addition, the potential height $W$ is assumed to be large enough so

$$
\exp \left(-\beta W_{1}\right) \ll 1, \quad \exp \left(-\beta W_{2}\right) \ll 1
$$

with

$$
W_{i}=\frac{L-a-\delta_{i}}{L-a} W \quad(i=1,2)
$$

are satisfied. It is evident that $\exp (-\beta W) \ll 1$ is satisfied if inequalities in Eq. (C5) hold. Under these assumptions, we have

$$
\alpha_{1} \simeq \alpha_{3} \simeq \frac{\beta W D_{0}}{L} \exp (-\beta W)
$$

and

$\beta_{0} \simeq \frac{\beta W D_{0}}{L} \exp \left(-\beta W_{1}\right), \quad \beta_{2} \simeq \frac{\beta W D_{0}}{L} \exp \left(-\beta W_{2}\right)$.

As for $\alpha_{0}, \alpha_{2}, \beta_{1}$, and $\beta_{3}$, we use the exact expressions given by Eqs. (B44) and (B13). In this way, some of the $\gamma$ 's defined in Eq. (B15) can be approximated as $\gamma_{0} \simeq \alpha_{0}, \gamma_{1} \simeq$ $\beta_{1}, \gamma_{2} \simeq \alpha_{2}$, and $\gamma_{3} \simeq \beta_{3}$, while we obtain $\gamma_{4}=\gamma_{5}=L / 2$ without approximation from Eq. (B52) for $F=0$. Similar approximations for $\xi$ 's and $\eta$ 's defined in Eqs. (B48) and (B49) can be made,

$$
\begin{gathered}
\xi_{0}^{1} \simeq \xi_{1}^{1} \simeq \frac{a L}{(\beta W)^{2} D_{0}} \exp (\beta W), \\
\eta_{0}^{1} \simeq \frac{L(L-a)}{(\beta W)^{2} D_{0}} \exp \left(\beta W_{1}\right), \\
\eta_{1}^{1} \simeq \frac{L(L-a)}{(\beta W)^{2} D_{0}} \exp \left(\beta W_{2}\right) .
\end{gathered}
$$

Now we are ready to write down approximate expressions for coefficients $C_{l m n}$ that appear in Eq. (30) for $R$ and are 
defined by Eqs. (B23)-(B30). Perhaps it is worth noting that we have

$$
\psi_{0} \frac{\gamma_{1}}{\gamma_{0}}+\psi_{1} \simeq \psi_{2} \frac{\gamma_{3}}{\gamma_{2}}+\psi_{3} \simeq \frac{L}{\beta W}
$$

under the condition (C5) and that

$$
\psi_{4} \frac{\gamma_{5}}{\gamma_{4}}+\psi_{5}=L
$$

holds exactly if $F=0$. From the approximate expressions presented above we obtain

$$
\begin{gathered}
C_{000} \simeq \frac{L}{\beta W}\left[\frac{(L-a)\left(1-\varepsilon_{2}\right)}{\beta W D_{0}} \frac{1}{\kappa_{\mathrm{T}}}+\frac{\varepsilon_{2}}{\kappa_{\mathrm{P}}}\right], \\
C_{100} \simeq \frac{L}{\kappa_{\mathrm{D}}}+\frac{(L-\delta) \delta}{2 D_{0}}+\frac{(L-a) \delta}{\beta W D_{0}}+\frac{L}{\beta W \kappa_{\mathrm{P}}}, \\
C_{010} \simeq \beta W \varepsilon_{1} C_{000}, \\
C_{110} \simeq \frac{L(L-a)}{\beta W D_{0}}\left(1-\varepsilon_{1}\right),
\end{gathered}
$$

for $C_{l m n}$ with $n=0$, where $\varepsilon_{i}(i=1,2)$ are defined in Eq. (43) in the text. The rest of $C_{l m n}$ are approximated as follows:

$$
\begin{gathered}
C_{001} \simeq \frac{L \varepsilon_{2}}{\beta W}\left[\frac{(L-\delta) \delta}{L D_{0}}+\frac{1}{\kappa_{\mathrm{D}}}\right], \\
C_{101} \simeq C_{001} / \varepsilon_{2}, \\
C_{011} \simeq \frac{L \varepsilon_{1}}{\beta W \kappa_{\mathrm{T}}}+\frac{L(L-a)\left(\varepsilon_{1}+\varepsilon_{2}\right)}{(\beta W)^{2} D_{0}} \\
-\frac{\varepsilon_{1} \varepsilon_{2}}{D_{0}}\left[\frac{(L-a) \delta}{\beta W}+\frac{2 L(L-a)}{(\beta W)^{2}}+\frac{(L-\delta) \delta}{2}\right], \\
C_{111} \simeq \frac{L(L-a)\left(1-\varepsilon_{1}\right)}{(\beta W)^{2} D_{0}} .
\end{gathered}
$$

If we substitute the parameter values given in Sec. III B into these expressions for $C_{l m n}$, we obtain $C_{001} / C_{000} \sim 0.01$, $C_{101} / C_{100} \sim 0.02, C_{011} / C_{010} \sim 0.05$, and $C_{111} / C_{110} \sim 0.09$. These values, together with the fact that $\rho_{\mathrm{P}}<1.1 \times 10^{-5}$ for $\left[\mathrm{P}_{\mathrm{i}}\right]<100 \mathrm{mM}$ (see Sec. III B), indicate that, among eight terms in Eq. (30) for $R, C_{001} \rho_{\mathrm{P}}, C_{101} \rho_{\mathrm{T}} \rho_{\mathrm{P}}, C_{011} \rho_{\mathrm{D}} \rho_{\mathrm{P}}$, and $C_{111} \rho_{\mathrm{T}} \rho_{\mathrm{D}} \rho_{\mathrm{P}}$ can be neglected compared to $C_{000}, C_{100} \rho_{\mathrm{T}}$, $C_{010} \rho_{\mathrm{D}}$, and $C_{110} \rho_{\mathrm{T}} \rho_{\mathrm{D}}$, respectively. In other words, the terms containing $\rho_{\mathrm{P}}$ can be neglected. Substitution of $R$ approximated in this way into Eq. (C4) yields Eq. (36) in the text.

\section{Velocity for $\boldsymbol{F}=\mathbf{0}$}

Let us investigate $Z_{v}$ defined in Eq. (32) in the case of $F=0$. The coefficients $\lambda_{\text {lmn }}$ in this equation are defined in Eqs. (B32)-(B39). Since function $G(F)$ appearing in these equations and defined in Eq. (B40) is zero if $F=0$, the coefficients other than $\lambda_{100}$ and $\lambda_{011}$ vanish in this case: $Z_{v}=\lambda_{100} \rho_{\mathrm{T}}+\lambda_{011} \rho_{\mathrm{D}} \rho_{\mathrm{P}}$. In addition, we have Eq. (C1), $\alpha_{3} / \beta_{2}=\beta_{3} / \alpha_{2}=\exp \left(u_{1}^{0}\right)$, and $\alpha_{4}=\beta_{5}$ for $F=0$ as well as identity $\gamma_{4}=\gamma_{5}$ [Eq. (B52)]; from these relations we obtain $\lambda_{011}=-\lambda_{000} \sigma$ with $\sigma$ given in Eq. (C2) and, hence, $Z_{v}=\lambda_{100}\left(\rho_{\mathrm{T}}-\sigma \rho_{\mathrm{D}} \rho_{\mathrm{P}}\right)$. Comparison of the last equation with
Eq. (31) yields

$$
\frac{v}{r}=\frac{Z_{v} L}{Z_{r}}=\frac{\gamma_{0} \alpha_{2} \alpha_{4}-\gamma_{0} \beta_{2} \beta_{4}-\beta_{0} \gamma_{2} \gamma_{4}}{\gamma_{0} \gamma_{2} \gamma_{4}} L
$$

for $F=0$. Note that the right-hand side in Eq. (C23) is independent of the nucleotide concentrations. This means that the velocity $v$ and the reaction rate $r$ show precisely the same dependence on the nucleotide concentrations if $F=0$ in the present model.

If the potential height $W$ is so large that the conditions in Eq. (C5) are satisfied, the second and third terms in the numerator in Eq. (C23) are negligibly small compared to the first term. Furthermore, we have $\gamma_{2} \simeq \alpha_{2}$ (see Sec. C 1), $\alpha_{4}=D_{0} /(L-\delta)$, and $\gamma_{4}=L D_{0} /(L-\delta) \delta$. Substituting these relations into Eq. (C23), we obtain Eq. (52) in the text.

\section{Velocity for small $\boldsymbol{F}$ and large $\boldsymbol{W}$}

In the preceding subsection, we have discussed $\lambda_{\operatorname{lm} n}$ in the case of $F=0$. Here, we calculate the first-order corrections to these coefficients in $F$ for small $F$. We assume that the conditions in Eq. (C5) are satisfied ( $\beta W$ is large). Then, by using the approximate calculations similar to the ones presented in Sec. C 1, the following results can be obtained for $\lambda_{\text {lmn }}$ with $n=0$ :

$$
\begin{aligned}
\lambda_{000} & \simeq-\varepsilon C_{000}\left(\beta F D_{0} / L\right), \\
\lambda_{100} \simeq \delta / L-\tau_{\mathrm{D}}\left(\beta F D_{0} / L\right), & \\
\lambda_{010} & \simeq-C_{010}\left(\beta F D_{0} / L\right), \\
\lambda_{110} & \simeq-C_{010}\left(\beta F D_{0} / L\right),
\end{aligned}
$$

where $C_{l m n}$ are approximate ones given in Sec. C 1 and $\tau_{\mathrm{D}}$ is defined in Eq. (44) and $\varepsilon$ by

$$
\varepsilon=(\beta W)^{2} \exp (-\beta W)
$$

The approximate expressions for $\lambda_{\operatorname{lm} n}$ with $n=1$ are given by

$$
\begin{aligned}
\lambda_{001} & \simeq-\varepsilon C_{001}\left(\beta F D_{0} / L\right), \\
\lambda_{101} & \simeq-\varepsilon C_{101}\left(\beta F D_{0} / L\right), \\
\lambda_{011} & \simeq\left(\varepsilon_{1} \varepsilon_{2} \delta / L\right)(1+\beta F \delta), \\
\lambda_{111} & \simeq-\varepsilon C_{111}\left(\beta F D_{0} / L\right),
\end{aligned}
$$

where $\varepsilon_{i}(i=1,2)$ are defined in Eq. (43) in the text and $C_{l m n}$ are those given in Sec. C 1. Substituting the parameter values given in Sec. III B into these expressions for $\lambda_{l m n}$, we find that the terms containing $\rho_{\mathrm{P}}$ in $Z_{v}$ are negligibly small if $\rho_{\mathrm{P}}<10^{-5}$ (which is appropriate for KIF1A as explained in Sec. III B) and condition (C3) is satisfied. Since we have $Z_{r} \simeq \rho_{\mathrm{T}}$ under the same condition, we obtain Eq. (55) in the text.

\section{APPENDIX D: POSSIBILITY OF ATP SYNTHESIS}

If the rate $r$ of ATP hydrolysis is negative $(r<0)$, the motor synthesizes ATP. From the expression (29) for $r$ together with Eqs. (31) and (34) we obtain

$$
\Delta \mu<\frac{W \delta}{L-a}+k_{\mathrm{B}} T \ln \sigma
$$



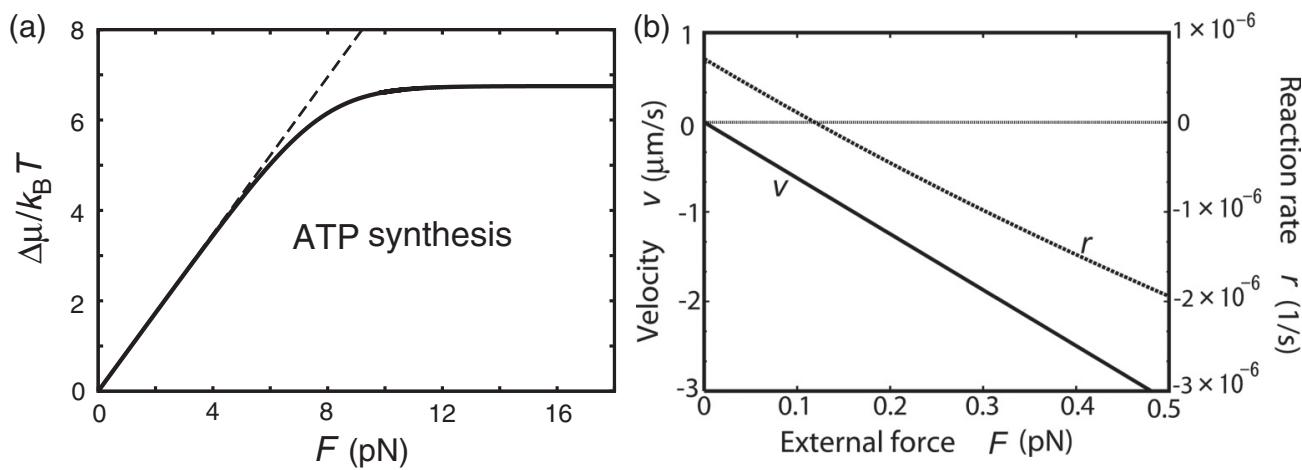

FIG. 12. (a) The region in the $(F, \Delta \mu)$ plane where the condition (D1) for the ATP synthesis is satisfied. The boundary of the region is represented by the solid line, whereas the dotted line corresponds to Eq. (D2). The boundary line approaches the horizontal line $\Delta \mu=$ $W \delta /(L-a)=6.75 k_{\mathrm{B}} T$ in the limit of $F \rightarrow \infty$. (b) The dependence of the velocity $v$ (solid line) and the reaction rate $r$ (dotted line) on the external force $F$ for $[\mathrm{ATP}]=1.0 \mathrm{nM},[\mathrm{ADP}]=21 \mathrm{mM}$, and $\left[\mathrm{P}_{\mathrm{i}}\right]=21 \mathrm{mM}$.

for the condition of ATP synthesis, where $\sigma$ defined in Eq. (B31) depends on the force $F$ but not on the nucleotide concentrations. The nucleotide concentrations enter into this condition only through $\Delta \mu$. Figure 12(a) shows the region in the $(F, \Delta \mu)$ plane where the condition (D1) for the ATP synthesis is satisfied. Note that there is an upper bound $W \delta /(L-a)=6.75 k_{\mathrm{B}} T$ to $\Delta \mu$ for the ATP synthesis to occur. The boundary line in Fig. 12(a) becomes a straight line for small $F$. This line can be approximately given by

$$
\Delta \mu \simeq \delta F,
$$

if the first condition in Eq. (35) is satisfied. The dotted line in Fig. 12(a) represents this approximate equation.

We see from Fig. 12(a) that $\Delta \mu$ has to be quite small for the ATP synthesis to occur with the force $F$ comparable to the stall force $\sim 0.15 \mathrm{pN}$ observed in Ref. [13]. To clarify whether the ATP synthesis can be measured experimentally we show the dependence of $r$ and velocity $v$ on the force $F$ in Fig. 12(b) for nucleotide concentrations $[\mathrm{ATP}]=1.0 \mathrm{nM},[\mathrm{ADP}]=21 \mathrm{mM}$, and $[\mathrm{Pi}]=21 \mathrm{mM}$, which corresponds to $\Delta \mu \simeq 0.1 k_{\mathrm{B}} T$. In this example we have $v \sim-2 \mu \mathrm{m} / \mathrm{s}$ and $r \sim-1.0 \times 10^{-6} \mathrm{~s}^{-1}$ for $F \sim 0.3 \mathrm{pN}$. This means that we need to wait about $10^{6}$ s (12 days) until KIF1A synthesizes a single ATP molecule. Meanwhile we have to pull KIF1A by distance of about 2 $\mathrm{m}$, which is much longer the mean run length $(\sim 1 \mu \mathrm{m})$ in the absence the external force. This result indicates that it is practically impossible to observe the ATP synthesis by KIF1A.

\section{APPENDIX E: APPROXIMATE EXPRESSIONS FOR ENERGETIC PROPERTIES}

Here we present approximate expressions for energetic properties $\eta^{*}, p^{*}, \eta^{\mathrm{g}}$ discussed in Sec. $\mathrm{V}$ under the condition (35) of far from equilibrium. We also discuss the conditions for the concentrations of ATP and ADP to realize the optimal values of these quantities.

\section{The maximum power $p^{*}$}

The maximum power $p^{*}$ for a given set of nucleotide concentrations $\rho_{\mathrm{T}}, \rho_{\mathrm{D}}$, and $\rho_{\mathrm{P}}$ is approximately given by

$$
p^{*} \simeq \frac{F_{\mathrm{s}} r \delta}{4}
$$

under the condition (35), as explained in Sec. V B. Under the same condition the stall force $F_{\mathrm{s}}$ can be approximated by Eq. (56), i.e.,

$$
F_{\mathrm{s}} \simeq \frac{\delta}{\beta D_{0}} \frac{\rho_{\mathrm{T}}}{C_{0}^{\prime}+\tau_{\mathrm{D}} \rho_{\mathrm{T}}+C_{\mathrm{D}} \rho_{\mathrm{D}}+C_{\mathrm{TD}} \rho_{\mathrm{T}} \rho_{\mathrm{D}}}
$$

and the reaction rate $r$ by

$$
r \simeq \frac{\rho_{\mathrm{T}}}{C_{0}+C_{\mathrm{T}} \rho_{\mathrm{T}}+C_{\mathrm{D}} \rho_{\mathrm{D}}+C_{\mathrm{TD}} \rho_{\mathrm{T}} \rho_{\mathrm{D}}},
$$

which is obtained by substituting Eqs. (37) and (38) into Eq. (36). It is noted that $p^{*}$ is independent of the phosphate concentration $\rho_{\mathrm{P}}$ in this approximation since expressions (E2) and (E3) for $F_{\mathrm{S}}$ and $r$ are independent of $\rho_{\mathrm{P}}$. Now it is clear from Eqs. (E2) and (E3) that both $F_{\mathrm{s}}$ and $r$ increase monotonically with $\rho_{\mathrm{T}}$ for a fixed valued of $\rho_{\mathrm{D}}$, whereas they decrease monotonically with increasing $\rho_{\mathrm{D}}$ for a fixed valued of $\rho_{\mathrm{T}}$. From these facts and expression (E1) for $p^{*}$, we see that the upper limit $p_{\text {max }}^{*}$ of $p^{*}$ is obtained by setting $\rho_{\mathrm{T}} \rightarrow \infty$ and $\rho_{\mathrm{D}}=0$, which results in

$$
p_{\max }^{*}=\frac{\delta^{2}}{4 \beta D_{0} \tau_{\mathrm{D}} C_{\mathrm{T}}} .
$$

\section{The maximum thermodynamic efficiency $\eta^{*}$ and the generalized efficiency $\eta^{\mathrm{g}}$}

The maximum thermodynamic efficiency $\eta^{*}$ for a given set of nucleotide concentrations is approximately given by Eq. (59), i.e., $\eta^{*} \simeq F_{\mathrm{s}} \delta / 4 \Delta \mu$ under the condition (35). Substituting expressions (E2) for $F_{\mathrm{s}}$ and (34) for $\Delta \mu$ into Eq. (59), we have

$\eta^{*} \simeq \frac{\delta^{2}}{4 D_{0} S(x)} \frac{\sqrt{x}}{\tau_{\mathrm{D}} \sqrt{x}+C_{\mathrm{D}} / \sqrt{x}+C_{\mathrm{TD}} \sqrt{y}+C_{0}^{\prime} / \sqrt{y}}$,

where

$$
x=\rho_{\mathrm{T}} / \rho_{\mathrm{D}}, \quad y=\rho_{\mathrm{T}} \rho_{\mathrm{D}},
$$

and

$$
S(x)=\ln \left(x / \rho_{\mathrm{P}}\right)+\beta W \delta /(L-a) .
$$

The maximum efficiency $\eta^{*}$ depends weakly (logarithmically) on $\rho_{\mathrm{P}}$ through function $S(x)$. Here, we are interested in the 
maximum of $\eta^{*}$ as a function of $\rho_{\mathrm{T}}$ and $\rho_{\mathrm{D}}$ for a fixed value of $\rho_{\mathrm{P}}$. In Eq. (E5) the dependence of $\eta^{*}$ on $\rho_{\mathrm{T}}$ and $\rho_{\mathrm{D}}$ is expressed as the dependence on $x$ and $y$ defined in Eq. (E6).

We, first, note that for a fixed value of $x$ the maximum of $\eta^{*}$ as a function of $y$ occurs at

$$
y=C_{0}^{\prime} / C_{\mathrm{TD}},
$$

which is independent of $x$. After substituting Eq. (E8) into Eq. (E5), we obtain the condition $d \eta^{*} / d x=0$ for $x$ to optimize $\eta^{*}$ as

$$
S(x)\left(\sqrt{\frac{C_{0}^{\prime} C_{\mathrm{TD}}}{x}}+\frac{C_{\mathrm{D}}}{x}\right)-2 \sqrt{\frac{C_{0}^{\prime} C_{\mathrm{TD}}}{x}}-\tau_{\mathrm{D}}-\frac{C_{\mathrm{D}}}{x}=0 .
$$

This equation for $x$ can be solved numerically. From $x$ and $y$ determined by Eqs. (E9) and (E8), respectively, the concentrations $\rho_{\mathrm{T}}$ and $\rho_{\mathrm{D}}$ that optimize $\eta^{*}$ are calculated by using Eq. (E6). Some of the results thus obtained are presented in Table I in the text.

The generalized efficiency $\eta^{\mathrm{g}}$ is defined by Eq. (61), i.e., $\eta^{\mathrm{g}}=r(0) \delta^{2} / \beta D_{0} \Delta \mu$. Under the condition (35), $r(0)$ can be approximated by Eq. (E3). Substituting this expression for $r$ and Eq. (34) for $\Delta \mu$ into Eq. (61), we have

$$
\eta^{\mathrm{g}} \simeq \frac{\delta^{2}}{D_{0} S(x)} \frac{\sqrt{x}}{C_{\mathrm{T}} \sqrt{x}+C_{\mathrm{D}} / \sqrt{x}+C_{\mathrm{TD}} \sqrt{y}+C_{0} / \sqrt{y}} .
$$

This equation is quite similar to Eq. (E5) for $\eta^{*}$ : If we replace $D_{0}$ by $4 D_{0}, C_{\mathrm{T}}$ by $\tau_{\mathrm{D}}$, and $C_{0}$ by $C_{0}^{\prime}$ in Eq. (E10), we obtain the expression for $\eta^{*}$. Therefore, the values of $x$ and $y$ that optimize $\eta^{\mathrm{g}}$ are obtained from Eqs. (E9) and (E8) with $C_{0}^{\prime}$ and $\tau_{\mathrm{D}}$ replaced, respectively, by $C_{0}$ and $C_{\mathrm{T}}$. The numerical values presented in the parentheses in Table II are obtained in this way.
[1] B. Alberts, A. Johnson, J. Lewis, M. Raff, K. Roberts, and P. Walter, Molecular Biology of the Cell, 4th ed. (Garland Science, New York, 2002).

[2] J. Howard, Mechanics of Motor Proteins and the Cytoskeleton (Sinauer Associates, Sunderland, MA, 2001).

[3] Molecular Motors, edited by M. Schliwa (Wiley-VCH, Weinheim, 2003).

[4] N. Hirokawa, R. Nitta, and Y. Okada, Nat. Rev. Mol. Cell Biol. 10, 877 (2009).

[5] N. Hirokawa, Y. Noda, Y. Tanaka, and S. Niwa, Nat. Rev. Mol. Cell Biol. 10, 682 (2009).

[6] Y. Okada and N. Hirokawa, Science 283, 1152 (1999).

[7] R. D. Astumian, Science 427, 917 (1997).

[8] F. Jülicher, A. Ajdari, and J. Prost, Rev. Mod. Phys. 69, 1269 (1997).

[9] C. Bustamante, D. Keller, and G. Oster, Acc. Chem. Res. 34, 412 (2001).

[10] P. Reimann, Phys. Rep. 361, 57 (2002).

[11] R. D. Astumian, Condens. Matter 17, S3753 (2005).

[12] Y. Okada and N. Hirokawa, Proc. Natl. Acad. Sci. USA 97, 640 (2000).

[13] Y. Okada, H. Higuchi, and N. Hirokawa, Nature 424, 574 (2003).

[14] K. Sasaki, R. Kanada, and S. Amari, J. Phys. Soc. Jpn. 76, 023003 (2007).

[15] I. Derényi, M. Bier, and R. D. Astumian, Phys. Rev. Lett. 83, 903 (1999).
[16] H. Wang and G. Oster, Europhys. Lett. 57, 134 (2002).

[17] A. Parmeggiani, F. Jülicher, A. Ajdari, and J. Prost, Phys. Rev. E 60, 2127 (1999).

[18] C. K. Bagdassarian and R. D. Astumian, in Thermodynamics in Biology, edited by E. di Cera (Oxford University Press, New York, 2000).

[19] R. Lipowsky, Phys. Rev. Lett. 85, 4401 (2000).

[20] R. Lipowsky and N. Jaster, J. Stat. Phys. 110, 1141 (2003).

[21] H. J. Woo and C. L. Moss, Phys. Rev. E 72, 051924 (2005).

[22] H. J. Woo, Phys. Rev. E 74, 011907 (2006).

[23] Although the reaction rate $r$ was not measured in the motility experiment in Ref. [13], the analysis of the forcevelocity relationship presented there implies that $r \sim 60 \mathrm{~s}^{-1}$ for $[\mathrm{ATP}]=2 \mathrm{mM}$.

[24] M. Moyer, S. Gilbert, and K. Johnson, Biochemistry 37, 800 (1998).

[25] S. Toyabe, T. Okamoto, T. Watanabe-Nakayama, H. Taketani, S. Kudo, and E. Muneyuki, Phys. Rev. Lett. 104, 198103 (2010).

[26] M. Bier and R. D. Astumian, Bioelectrochem. Bioenerg. 39, 67 (1996).

[27] R. Kanada, T. Kuwata, H. Kenzaki, and S. Takada, PLoS Comput. Biol. 9, e1002907 (2013).

[28] T. C. Elston, D. You, and C. S. Peskin, SIAM J. Appl. Math. 61, 776 (2000).

[29] K. B. Zeldovich, J.-F. Joanny, and J. Prost, Eur. Phys. J. E. 17, 155 (2005).

[30] P. Sanker, Physica A 359, 85 (2006). 